\title{
Modeling far-infrared emission from the HII region S125
}

\author{
P. A. Aannestad ${ }^{1}$ and R. J. Emery ${ }^{2}$ \\ 1 Department of Physics and Astronomy, Arizona State University, Tempe, AZ 85287-1504, USA \\ 2 Space Science Department, Rutherford Appleton Lab, Chilton, Oxon OX11 0QX, UK
}

Received 30 March 2001 / Accepted 5 July 2001

\begin{abstract}
We present ISO-LWS $45-180 \mu \mathrm{m}$ spectral observations at 17 positions in the HII region S125. We model, as one integrated region, the far-IR continuum emission as well as the observed emissions in the radio continuum and in the $21 \mathrm{~cm}$ line, comparing two different model density distributions and several silicate-carbon dust models. The dust in the HII region is found to be severely depleted, while the dust in the PDR region has a normal dust/gas ratio, but with a major fraction of the mass in the form of the very small dust grains. Silicatecarbon dust must have strongly enhanced far-IR emissivities to be consistent with the observed spectra. It is found that the ionizing star alone does not power the infrared luminosity, but that additional sources of far-IR radiation must be present in a ring-like region surrounding the HII region. In this region the star accounts for about $50 \%$ of the observed emission. For most positions, the additional emission peaks at a wavelength around $70 \mu \mathrm{m}$.
\end{abstract}

Key words. HII regions - ISM: individual objects: S125 - ISM: dust, extinction - infrared: ISM

\section{Introduction}

HII regions provide important information about the interaction of young, massive stars with their surrounding gas and dust. The stellar UV radiation dissociates and ionizes the gas and heats the dust, the dust re-emitting the absorbed energy as infrared radiation. The dust sizes and composition may be modified by the UV radiation, and radiation pressure on the dust can be dynamically important. If a massive star forms in the outer parts of a molecular cloud, where a strong density gradient is present, a "blister" HII region forms, in which the ionization front "opens up" in the direction of lower density. In the direction of higher density, beyond the ionization front, a photodissociation region (PDR) is formed, where $\mathrm{H}_{2}$ molecules are dissociated by UV photons and reformed on the surfaces of the dust grains. The structure of the PDR is thus strongly dependent on the amount and nature of the nebular dust, which manifests itself mainly through its emitted infrared radiation. By studying the dust emission we then gain insight into not only the nature of the dust, but also the morphology of the HII region/PDR as a whole, as well as tracking the properties of the stellar radiation field.

The HII region S125 (IC 5146) appears to be a prototype blister seen face-on and on the near side of a parent molecular cloud. It is a highly symmetric emission nebula, about $0.2^{\circ}$ in diameter and ionized by a single star $\mathrm{BD}+46^{\circ} 3474$ at a distance from the Sun of $960 \mathrm{pc}$

Send offprint requests to: P. A. Aannestad,

e-mail: per.aannestad@asu.edu
(Crampton \& Fisher 1974). A detailed model was presented by Roger \& Irwin (1982, herafter RI), who reproduced observations of both the HII radio continuum emission and the HI line emission. Enhanced CO emission has been observed around the periphery of S125 (Lada \& Elmegreen 1979; McCutcheon et al. 1982; Dobashi et al. 1992), and Sargent et al. (1981) mapped the region at 85 and $150 \mu \mathrm{m}$ with a $4.5^{\prime}$ beam. The far-IR maps indicated the presence of at least one embedded protostar, but much of the dust heating was attributed to the exciting star in S125.

RI observed streaming of ionized gas in the direction of lower density at velocities $2-10 \mathrm{~km} \mathrm{~s}^{-1}$, implying an "evaporative flow" off the denser, neutral material and setting a timescale for the development of this part of the region. Our emphasis is on finding the present density distribution and present properties of gas and dust. Also, we only consider the dust infrared continuum emission here. Observations of fine-structure lines of OI and CII will be dealt with in a forthcoming paper.

We present 18 ISO-LWS far-IR spectra of S125 as observed at equal intervals along two perpendicular scans across the center of the nebula, spanning an angular width of about $0.4^{\circ}$ and using a beamwidth of $1.3^{\prime}$. We model this emission as well as the existing HII and HI data, starting with the RI spherically symmetric density distribution and then introducing an axisymmetric blister model. Requiring the model to account for the radio continuum from the HII region, the HI line emission from the PDR, as well as the far-IR dust emission from both the 
ionized and the $\mathrm{HI} / \mathrm{H}_{2}$ neutral regions, adds significant extra constraints on the previous modeling work. While S125 appears free of many of the complexities often found in star-forming regions, the ionizing star alone does does not appear to power the infrared luminosity and our modeling helps to define the additional components that need to be considered.

In Sect. 2 we outline our modeling of the HII region, while Sect. 3 describes our treatment of the PDR, including a detailed treatment of the $\mathrm{H}_{2}$ formation and destruction rates. Section 4 describes the silicate-carbon dust models employed, which include enhancements of the farIR emissivity due to composition or shape. The LWS spectra are presented in Sect. 5, whilst Sect. 6 presents modeling and comparison of HII, HI and IR emission within the context of the RI geometry, but with more realistic dust absorption properties. We also investigate the transition region $2 \mathrm{H} \rightarrow \mathrm{H}_{2}$, and how it depends on common approximations to the formation and destruction rates of $\mathrm{H}_{2}$. In Sect. 7 we subject a blister model density distribution to the same constraints as the RI model. Discussion is in Sect. 8, and conclusions are given in Sect. 9.

\section{Modeling the HII region}

The ionization equlibrium of $\mathrm{H}, \mathrm{He}, \mathrm{O}$, and $\mathrm{N}$ is calculated as in our earlier work on two-dimensional blister models (Aannestad \& Emery 1986; Aannestad 1989). In addition we here include $\mathrm{C}$ ionization up to CIV. Photoionization cross sections for all the elements are taken as the analytic fits given by Verner et al. (1996). The charge exchange rates of C ions with $\mathrm{H}$ are from Kingdon \& Ferland (1996), and for exchanges between CIII, CIV and He the rates are from Butler \& Dalgarno (1980) and Arnaud \& Rothenflug (1985).

The ionizing star in $\mathrm{S} 125$ is the B0V star BD $+46^{\circ} 3474$ (Crampton \& Fisher 1974). We adopt the radiation field of a stellar atmosphere NLTE calculation by Aufdenberg (1998, private communication) at an effective temperature of $30000 \mathrm{~K}, \log g$ of 4.0 , and assume a radius of $7.4 R_{\odot}$. The ionizing photon flux is $3.5 \times 10^{47}$ photons/s, significantly higher than the Kurucz (1993) LTE model flux of $1.9 \times 10^{47}$ photons/s. Most of the difference between the two model calculations is due to the effect of sphericity in the NLTE calculation (Aufdenberg et al. 1999) The ionizing luminosity is $9.3 \times 10^{36} \mathrm{erg} / \mathrm{s}$, and the nonionizing luminosity is $1.5 \times 10^{38} \mathrm{erg} / \mathrm{s}$, the latter being essentially the same for the LTE and NLTE atmosphere models. The models assumed solar abundances, but the lower B-star abundances (Gies \& Lambert 1992) have essentially no effect on the spectrum (Aufdenberg 2000, private communication).

The dust/gas ratios for the dust components are taken to be constant throughout the ionized region, except for a region surrounding the star. The volume of this region can be determined either by setting an upper limit to individual grain temperatures, or by assuming an inner "evacuated" region with no gas or dust. The far-infrared observations reported here require the latter approach (Sect. 6.1). Such a low-density region may have been formed by a stellar wind or by radiation pressure on the dust.

The radiative transfer for ionizing photons, including the opacity of $\mathrm{C}, \mathrm{N}$, and $\mathrm{O}$ ions, is solved in the "on-thespot outwards-only" approximation on a grid of 37 radial directions with up to 100 radial points for 49 frequency points of ionizing photons between 0.023 and $0.091 \mu \mathrm{m}$. The electron temperature is assumed constant at a value of $7000 \mathrm{~K}$ (RI). The initial calculation assumes no dust evaporation and no scattering due to dust. A subsequent calculation then finds the dust evaporation boundaries (if required) and corrective factors for scattering (Aannestad 1989), as well as escape probabilities for $\mathrm{Ly}-\alpha$ photons to be used in the final calculation of the ionization and thermal dust structure. This solution to the HII region structure then provides the starting point for the PDR calculation.

\section{Modeling the PDR}

The radiation input to the $\mathrm{PDR}$ is given directly by the modelled radiation passing out of the HII region, through the ionization front. A basic assumption in the modeling here is that the infrared emission is optically thin. Thus, for the directions in which an ionization boundary is found, the calculation proceeds for non-ionizing photons (365 frequency points) until one of the following criteria is met: 1) the dust optical depth at $10 \mu \mathrm{m}$ reaches 0.5 ; or 2) the radial distance from the star is larger than $13.5 \mathrm{pc}$ for the RI density distribution, or 30 scale heights for the blister density distribution. In addition, for the RI model where the density has a maximum and then decreases, the calculation stops if the total number density falls below $1 \mathrm{~cm}^{-3}$. No account is taken of a possible radiation field on the "backside" of the cloud.

The dust grains in this region are assumed to have the same composition as the dust in the HII region, but may have different (constant) dust/gas ratios and size distributions. The radiation field and the (equilibrium) dust temperatures are calculated allowing only for the optical depth of the dust and $\mathrm{H}_{2}$ molecules (cf. Sect. 3.1). For the gas temperature, which enters into the calculation of the $\mathrm{H}_{2}$ formation rate, we assume a simple dependence on the dust temperatures and optical depth:

$T_{\mathrm{g}}\left(\tau_{\mathrm{d}}\right)=<T_{\mathrm{d}}\left(\tau_{\mathrm{d}}\right)>+\frac{\Delta T_{0}}{1+\tau_{\mathrm{d}}}$

where

$\Delta T_{0}=\left(T_{0}-<T_{\mathrm{d}, \mathrm{b}}>\right)\left(1+\tau_{\mathrm{d}, \mathrm{b}}\right)$.

Here $\tau_{\mathrm{d}}$ is the dust optical depth at $0.1 \mu \mathrm{m}, \tau_{\mathrm{d}, \mathrm{b}}$ is the same optical depth at the ionization boundary, $\left\langle T_{\mathrm{d}}>\right.$ and $\left\langle T_{\mathrm{d}, \mathrm{b}}\right\rangle$ are the average dust temperatures, and $T_{0}$ is an assumed gas temperature at the boundary, assumed here to be $T_{0}=500 \mathrm{~K}$, independent of direction from the star. A similar dependence upon optical depth was used 
by Draine \& Bertoldi (1996) for illustrative purposes, but without a coupling to the dust temperature at large optical depths. While Eq. (1) is not based on numerical calculations, it does reflect the expected behavior, and will suffice for our purposes. Levenson et al. (2000) recently found a temperature of about $900 \mathrm{~K}$ at the molecular cloud interface in M16. At such high temperatures chemical destruction of $\mathrm{H}_{2}$ could become important, but is not included here. At any case, the temperature falls rapidly away from the boundary (cf. Fig. 7), limiting the importance of chemical reactions. For the average dust temperature above, we calculate an average as weighted by the surface area of the grains:

$<T_{\mathrm{d}}>=\frac{\Sigma T_{\mathrm{d}}(a) n(a) a^{2}}{\Sigma n(a) a^{2}}$.

The summation is over size and composition. For the range of densities considered here $\left(n \sim 10^{2}-10^{3} \mathrm{~cm}^{-3}\right)$, we neglect any energy input to the dust grains from either gas particle encounters or molecular formation processes since the radiative input to the grains dominates their energy budget.

\section{1. $\mathrm{H}_{2}$ formation and destruction}

Molecular hydrogen forms on the surfaces of dust grains and is dissociated by far-ultraviolet photons in the 912-1110 A range. The rate of formation $\left(\mathrm{cm}^{-3} \mathrm{~s}^{-1}\right)$ can be written as a summation over all grains

$R_{\mathrm{form}}=\sum_{i} 2 \pi a_{i}^{2} n\left(a_{i}\right) S\left(a_{i}, T_{\mathrm{g}}\right) \gamma\left(a_{i}\right)\left(\frac{k T_{\mathrm{g}}}{2 \pi m_{\mathrm{H}}}\right)^{1 / 2} n(H)(4)$

where $n(\mathrm{H})$ is the number density of $\mathrm{H}$-atoms of mass $m_{\mathrm{H}}, S$ is the sticking efficiency of $\mathrm{H}$-atoms, and $\gamma(a)$ is the recombination efficiency of $\mathrm{H}+\mathrm{H} \rightarrow \mathrm{H}_{2}$. In order for recombination to be efficient, the atoms must not evaporate before having a chance to recombine, setting an upper limit on the grain temperature. Depending on the assumptions about the binding energies on the surface and the distribution of "enhanced" binding sites (Hollenbach \& Salpeter 1971), the limiting temperature $T_{\mathrm{r}}$ is in the range $70-30 \mathrm{~K}$. Here we adopt

$\gamma(a)=1$ if $T_{\mathrm{d}}(a) \leq 50 \mathrm{~K} \quad \gamma(a)=0$ if $T_{\mathrm{d}}(a)>50 \mathrm{~K} .(5)$

Biham et al. (1998) have pointed out that Eq. (4) strictly applies only in the limit of high recombination efficiency. In the other limit of low recombination efficiency (low mobility or low coverage), the rate of formation is quadratic rather than linear in the flux of incoming H-atoms. Experimental support for this dependence has been found by Pirronello et al. (1999) The details will depend on the energy barrier for $\mathrm{H}$-atom diffusion, which is poorly known for the surfaces pertaining to interstellar dust grains. By applying Eqs. (4)-(5) in the calculations here for relatively diffuse cloud densities we may be overestimating the $\mathrm{H}_{2}$ formation rate at the lowest dust temperatures, when the mobility of the atoms is the least.

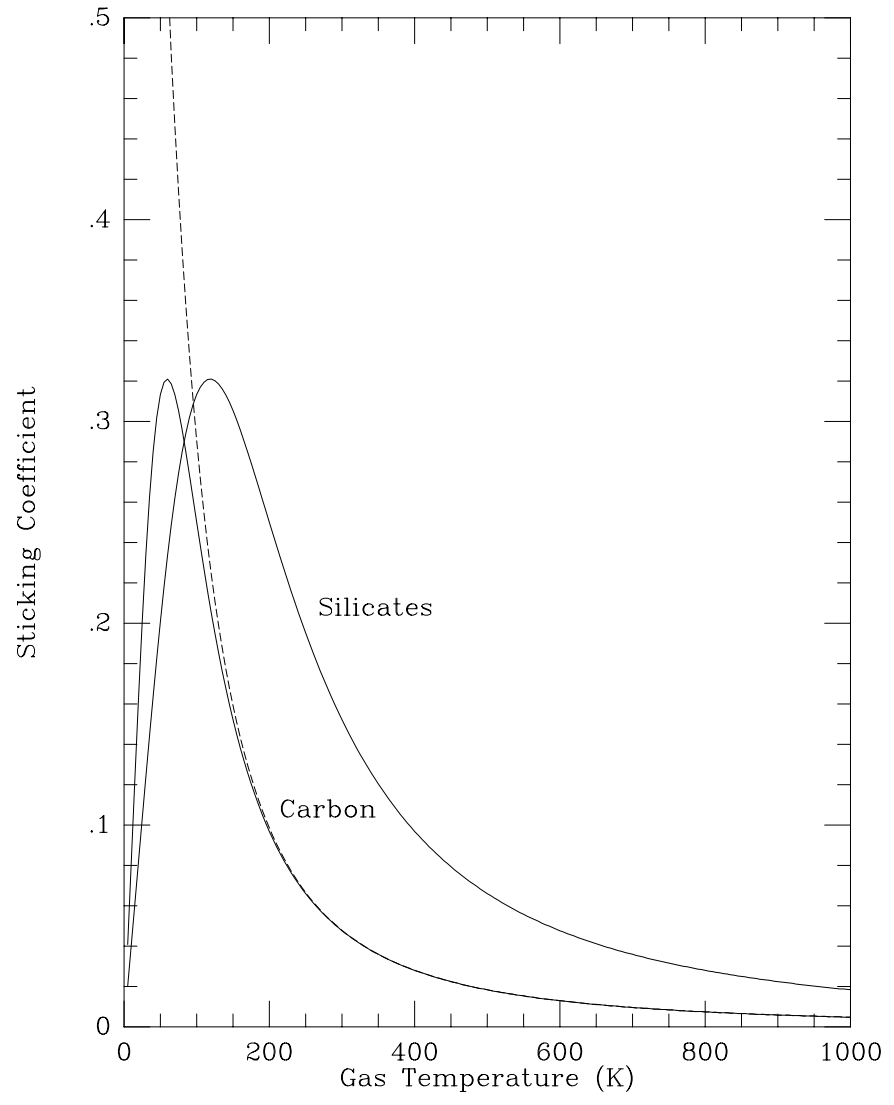

Fig. 1. The sticking coefficient for $\mathrm{H}$ atoms adsorbed on silicate or carbon surfaces as assumed in this work and adopted after Leitch-Devlin \& Williams (1985). The dashed curve shows the sticking coefficient of Hollenbach \& Salpeter (1971) using the carbon parameter.

For the sticking efficiency $S$ we adopt an expression dependent only on the gas temperature that reproduces the general behavior for graphite and oxides as calculated by Leitch-Devlin \& Williams (1985). The sticking coefficients are sensitive to the type of interaction with the surface and to the lattice phonon spectrum, but for the dust temperatures of interest here they show a general peak at the lower gas temperatures. This behavior differs from the work of Hollenbach \& Salpeter (1971) that gave a monotonic increase in $S$ with decreasing gas temperature. Defining $\Gamma=T_{\mathrm{c}} / T_{\mathrm{g}}$ the following expression

$S(\Gamma)=\frac{\Gamma^{2}+0.8 \Gamma^{3}}{2+2.4 \Gamma+\Gamma^{2}+0.8 \Gamma^{3}+\Gamma^{4}}$

is shown in Fig. 1, where we adopt $T_{\mathrm{c}}=100 \mathrm{~K}$ and $200 \mathrm{~K}$ for the carbon dust and silicate dust, respectively. For comparison the dashed curve shows the sticking coefficient of Hollenbach \& Salpeter (1971) for $T_{\mathrm{c}}=100 \mathrm{~K}$.

For the rate of dissociation $\left(\mathrm{cm}^{-3} \mathrm{~s}^{-1}\right)$ of $\mathrm{H}_{2}$ molecules of density $n\left(\mathrm{H}_{2}\right)$ by the radiation field we write (Bertoldi \& Draine 1996)

$R_{\text {diss }}=3.8 \times 10^{-18} n\left(\mathrm{H}_{2}\right) F_{\mathrm{uv}}\left(\tau_{\mathrm{d}}\right) f_{\mathrm{s}}\left(N_{\mathrm{H}_{2}}\right)$,

where $F_{\mathrm{uv}}\left(\tau_{\mathrm{d}}\right)$ is the photon flux $\left(\mathrm{cm}^{-2} \mathrm{~s}^{-1}\right)$ in the wavelength interval $912-1110 \AA$ as a function of the dust optical 
depth $\tau_{\mathrm{d}}$. The function $f_{\mathrm{s}}$ is the best approximation to the self-shielding as a function of the $\mathrm{H}_{2}$ column density $N_{\mathrm{H}_{2}}$ as given by Draine \& Bertoldi (1996; their Eq. (37), with a broadening parameter $\left.b_{5}=3 \mathrm{~km} \mathrm{~s}^{-1}\right)$. The effect of this dissociation on the photon flux as it heats the dust is taken into account by defining an optical depth $\tau_{\mathrm{H}_{2}}=-\ln f_{\mathrm{s}}$.

Assuming the equilibrium condition $R_{\text {form }}=R_{\text {diss }}$ at a radial position $p$, and defining the fraction $x \equiv$ $2 n\left(\mathrm{H}_{2}\right) / n_{\mathrm{t}}(p)$, where $n_{\mathrm{t}}$ is the total number of H-nuclei, we obtain

$3.8 \times 10^{-18} F_{\mathrm{uv}}(p) x f_{\mathrm{s}}\left(N_{\mathrm{H}_{2}}\right)-2(1-x) n_{\mathrm{t}}(p) R_{\mathrm{d}}(p)=0,(8)$

where

$N_{\mathrm{H}_{2}}=\int_{\mathrm{p}_{0}}^{p} \frac{1}{2} x n_{\mathrm{t}}(p) \mathrm{d} p$,

$p_{0}$ is the radial position at the PDR boundary, and $R_{\mathrm{d}} \equiv$ $R_{\text {form }} /\left(n(\mathrm{H}) n_{\mathrm{t}}\right)$. Equation (8) can be solved for $x$ by simple bi-section.

\section{Dust emission properties}

\subsection{Basic dust model}

The nature (composition, sizes, optical properties) of dust grains in the regions of star formation is uncertain. We shall consider a set of dust models, designed to cover reasonable variations in size ranges and optical properties, but assuming the basic composition of a silicate-carbon mixture. A silicate- graphite mixture similar to that of Mathis et al. (1977) with the "astronomical" silicate and graphite optical constants tabulated by Draine \& Lee (1984) is our reference model, since it is in good agreement with the mean interstellar extinction curve. However, owing to the quite cool emission in the observed spectra (Sect. 5), it is necessary to include models with substantially increased far-infrared emissivity. We shall also consider the possibility that the dust in the HII region may differ significantly in its infrared emissivities from the dust in the neutral region.

The size distribution of each component in the grain mixture is sampled by taking 30 values over the size range $0.0156 \mu \mathrm{m}$ to $0.93 \mu \mathrm{m}$, in addition to a set of "very small" dust grains, with properties calculated for spheres of radius $0.005 \mu \mathrm{m}$. A similar division into large and "very small" grains has recently been used by Li \& Draine (2001) in modeling the infrared emission spectra for various general parts of the Galaxy. They include PAH-like properties for the very small carbonaceous grains, which we do not. However, since in our case we only model the far-infrared emission, the details of the very small sized grains are relatively unimportant.

The upper and lower bounds in our grain size distributions can be varied separately for the carbon and silicate components, as well as between the HII region dust and the PDR dust. The total amount of dust material is set by specifying the fractional amounts of the cosmic abundances of Si and $\mathrm{C}$ that are present in the very small grains and the larger grains separately. For cosmic abundances we adopt the recent revision of the standard solar composition by Grevesse \& Sauval (1998).

There is at present uncertainty regarding the applicability of solar abundances to the interstellar medium. In particular, the $\mathrm{C}, \mathrm{N}$, and $\mathrm{O}$ abundances in B stars appear less than solar, contrary to expectation for metal enrichment as a simple function of age (Gies \& Lambert 1992) The B-star C/H ratio as given by Sofia et al. (1997) is $2.4 \pm 0.5 \times 10^{-4}$, or $76 \pm 15 \%$ of the carbon abundance adopted here. If this is typical of the general interstellar carbon abundance, it is difficult for current dust models to account for the amount of extinction per $\mathrm{H}$ nucleus. Mathis (1996) has discussed this problem and proposed composite spheroidal and fluffy grains as a way to solve the carbon "crisis". However, here we shall just assume that the (new) solar reference abundances are applicable to the S125 region and adopt spherical grains allowing for "fluffiness" as explained below.

The grain size distributions are assumed to follow the $a^{-3.5}$ power law distribution (Mathis et al. 1977). The cross-section $/ \mathrm{H}$-atom $\propto[\mathrm{X} / \mathrm{H}]_{\mathrm{d}} / \rho_{\mathrm{d}}$, where $\mathrm{X}$ is $\mathrm{C}$ or $\mathrm{Si}$ for carbon and silicate grains, respectively, and $\rho_{\mathrm{d}}$ is the material density. Often used values for $\rho_{\mathrm{d}}$ are $2.26 \mathrm{~g} \mathrm{~cm}^{-3}$ for graphite and $3.3 \mathrm{~g} \mathrm{~cm}^{-3}$ for silicates. Fluffy grains, however, may have lower densities, and we consider the values of 2.0 and $3.0 \mathrm{~g} \mathrm{~cm}^{-3}$, respectively. The optical properties of fluffy particles may of course be different from homogenous particles. Using the Maxwell-Garnett rule (Bohren \& Huffman 1983), the extinction properties of a slightly fluffy particle may be approximated. We have compared such calculations, assuming vacuum volume fractions of $10 \%$ and $20 \%$, with Mie calculations of the less dense homogenous particles, and find curves consistent with each other and with the observed average extinction values given by Savage \& Mathis (1979). This basic silicate-carbon dust model has lower and upper size limits for the large grains of $0.0156 \mu \mathrm{m}$ and $0.25 \mu \mathrm{m}$ for both types of grains, respectively. The fractions of the cosmic abundance of $\mathrm{C}$ in the dust are $[\mathrm{X} / \mathrm{H}]_{\mathrm{d}}=0.53$ and 0.12 for the large and very small graphite particles, respectively. For $\mathrm{Si}$ the same fractions are 0.9 and 0.08 . The carbon fraction in the dust leaves a fraction $1.16 \times 10^{-4}$ for the interstellar gas phase $\mathrm{C} / \mathrm{H}$ ratio, compared to the observational value $(1.4 \pm 0.2) \times 10^{-4}$ (Cardelli et al. 1996). The values of total to selective extinction $A_{V} / E(B-V)$ for the basic model calculations range from 2.95 to 3.09 , and the values of $N(\mathrm{H}) /(E(B-V)$ are in the range $(5.7-6.3) \times 10^{21} \mathrm{H} \mathrm{cm}^{-2} \mathrm{mag}^{-1}$. These compare well to the typical observed values of $3.1 \pm 0.1$ and $5.8 \times 10^{21} \mathrm{H} \mathrm{cm}^{-2} \mathrm{mag}^{-1}$ (Mathis 1996).

\subsection{Dust with enhanced IR emissivity}

In addition to the basic dust model considered above, we also include the case for grains with enhanced far- infrared emissivites. Laboratory investigations as well as 
astronomical data indicate that the far-infrared opacity law for small particles behaves as $\lambda^{-\beta}$ with $\beta$ in the range of 1-2 (see Weintraub et al. 1991; Pollack et al. 1994). The lower values of the spectral index $\beta$ apply to amorphous and more planar grain structures. Here we shall just assume $\beta=1.0$ for $\lambda>50 \mu \mathrm{m}$ for the silicate-carbon mixture.

\subsection{Dust with fractal structure}

A fractal structure for a dust grain may enhance its infrared absorption cross section significantly. Bazell \& Dwek (1990) showed that the enhancement could be up to a factor of 2 for silicates and a factor of 6 for amorphous carbon grains compared to spherical grains of radius $0.01 \mu \mathrm{m}$. The largest enhancements were for $\lambda>10 \mu \mathrm{m}$. The optical properties for amorphous carbon came from Edoh (1983). Our "fractal" dust model uses the astronomical silicates and the Edoh optical constants as listed by Hanner (1987), and assumes that the Bazell \& Dwek enhancement factors are the same for all our sizes. The material density of amorphous carbon is set to $1.5 \mathrm{~g} \mathrm{~cm}^{-3}$.

\subsection{Non-spherical dust grains}

As our last dust model we shall approximate the effect of shape. Nonspherical grains have larger absorption cross sections than spherical grains, as shown in the extensive investigation by Fogel \& Leung (1998). Here we simply assume the uniform distribution of ellipsoidal shapes (Bohren \& Huffman 1983) for the silicate - amorphous carbon mixture at all particle sizes.

Figure 2 shows the absorption opacity per H-atom for the basic dust model, the model with enhanced far infrared emissivities, the fractal model, and the nonspherical dust model. The fractions of carbon and silicon in the dust and the lower and upper limits for the size distributions are the same as in the the basic dust model.

\section{Observations}

ISO-LWS spectra in the range $45-196 \mu \mathrm{m}$, were obtained in a set of 17 positions indicated in Fig. 3, which is a $0.5^{\circ} \times 0.5^{\circ}$ image from the Digitized Sky Survey (SkyView Virtual Telecope). The pointings make up scans at $45^{\circ}$ with respect to a line of constant declination, with $3^{\prime}$ spacing, centered on the position $\mathrm{RA}(1950)=$ $21^{\mathrm{h}} 51^{\mathrm{m}} 37^{\mathrm{s}}, \delta(1950)=47^{\circ} 02^{\prime} 07^{\prime \prime}$. The approximately $80^{\prime \prime}$ $F W H M$ beam is also indicated. The ionizing star is at a position $0.68^{\prime}$ West and $0.29^{\prime}$ South of the central raster position.

Figures 4 and 5 show the LWS spectra for the two raster scans $\mathrm{B}-\mathrm{H}$, and $\mathrm{J}-\mathrm{R}$, respectively. For clarity in Fig. 4, the relatively noisy spectra of the outlier points A and I have been omitted. The scatter in the data of an individual spectrum is an indication of the systematic errors in the broad continuum shape that is compared with the model results.

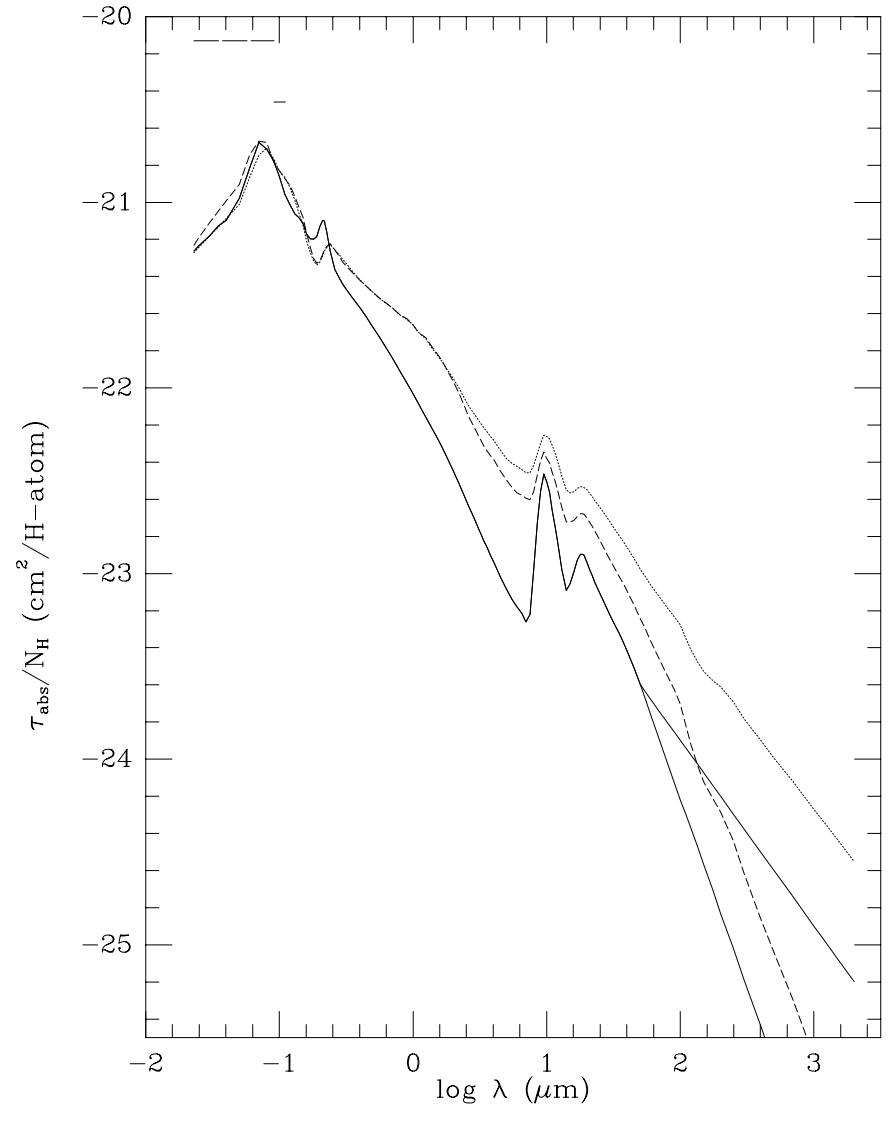

Fig. 2. Absorption cross section per $\mathrm{H}$ atom for silicate-carbon dust models. The solid curves are for the fluffy silicate-graphite dust, with and without an assumed $\lambda^{-1.0}$ behavior for $\lambda>$ $50 \mu \mathrm{m}$. The dashed curve is for assumed fractal grains of silicate and amorphous carbon, and the dotted curve is for a continuous distribution of ellipsoids of silicate and amorphous carbon. In all cases, the characteristic sizes are assumed to follow a $a^{-3.5}$ power law distribution from $0.0156-0.25 \mu \mathrm{m}$, plus a set of very small grains of size $0.005 \mu \mathrm{m}$. Also shown as horizontal dashed lines in the ultraviolet are the values assumed for the dust in the model of S125 by Roger \& Irwin (1982).

These spectra are in general very broad with a maximum in the $60-80 \mu \mathrm{m}$ range, with superposed line emission from OI at $63 \mu \mathrm{m}$ and CII at $158 \mu \mathrm{m}$ along some of the lines of sight. There is essentially no change in the shape of the spectrum across the central part of the nebula (positions D-F and $\mathrm{M}-\mathrm{O}$ ), and the center positions $(\mathrm{E}$ and $\mathrm{N}$ ) show a lower level of continuum emission than the neighbouring points. Given the near-central position of the star, this suggests both a central region depleted of dust and dust emission dominated by emission at temperatures around $30 \mathrm{~K}$. In our modeling, therefore, we include an "evacuated" region (no dust or gas) near the star as well as a very low dust/gas ratio in the HII region. The observed continuum emission spectra must primarily reflect the variation in the dust properties within the neutral gas. 


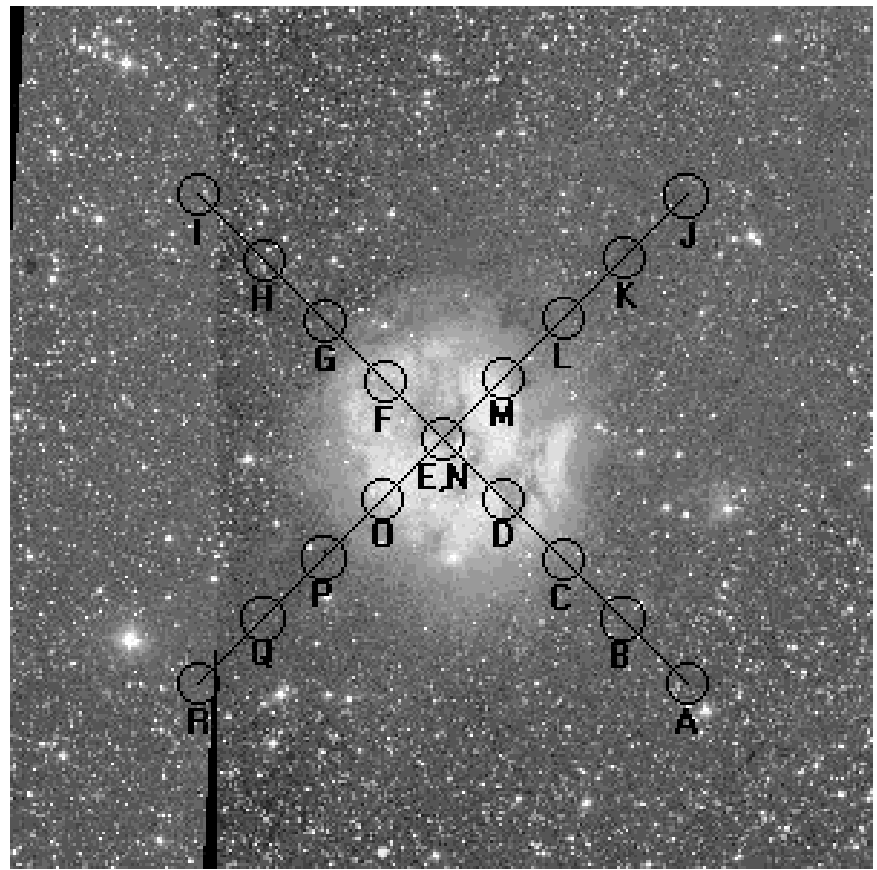

Fig. 3. Raster Scan positions for the LWS beam in a $0.5^{\circ} \times 0.5^{\circ}$ view centered on $\mathrm{RA}(1950)=21^{\mathrm{h}} 51^{\mathrm{m}} 37^{\mathrm{s}}, \delta(1950)=47^{\circ} 02^{\prime} 07^{\prime \prime}$. North is up and RA increases to the left.

\section{Model building on the RI geometry}

The model proposed by Roger \& Irwin (1982) (RI model) assumes a Gaussian density distribution for the associated molecular cloud with a peak density of 620 atoms cm $\mathrm{cm}^{-3}$ at $3 \mathrm{pc}$ from the ionizing star, an e-folding radius of $2.6 \mathrm{pc}$, and a "density reduction" factor in the HII region of 2.6 (giving a density of $62 \mathrm{~cm}^{-3}$ at the star). It is constrained not only by the observed emission measure as a function of the projected radius, but also by the observed mean profile of $21 \mathrm{~cm}$ emission from $\mathrm{HI}$ in the PDR. The model used the density reduction factor as a free parameter to mimic changes in either the photon flux or the dust absorption in the HII region. The dust absorption coefficient was assumed to be independent of wavelength with values of $7.4 \times 10^{-21} \mathrm{~cm}^{2} / \mathrm{H}$-atom and $3.5 \times 10^{-21} \mathrm{~cm}^{2} / \mathrm{H}$-atom for $\mathrm{H}$-ionizing photons and $\mathrm{H}_{2}$-dissociating photons, respectively (cf. Fig. 2). The $\mathrm{H}_{2}$ formation rate coefficient $R_{\mathrm{d}}$ in the PDR was also assumed constant, with a best-fit value of $3.5 \times 10^{-17} \mathrm{~cm}^{3} \mathrm{~s}^{-1}$.

\subsection{Model results for the HII region}

Following RI, we attempt to fit the observed distribution of the emission measure, or equivalently the brightness temperature at $1.42 \mathrm{GHz}$, (RI Fig. 3). We adopt the same density distribution and placement of the star as RI, except for a slightly smaller peak density of $610 \mathrm{~cm}^{-3}$ and a smaller "density reduction" factor of 1.8. Because of the lack of significant far-infrared emission from dust in the HII region (Sect. 5), the dust/gas mass ratio is set to $0.75 \times 10^{-6}$, so that there is very little absorption by dust

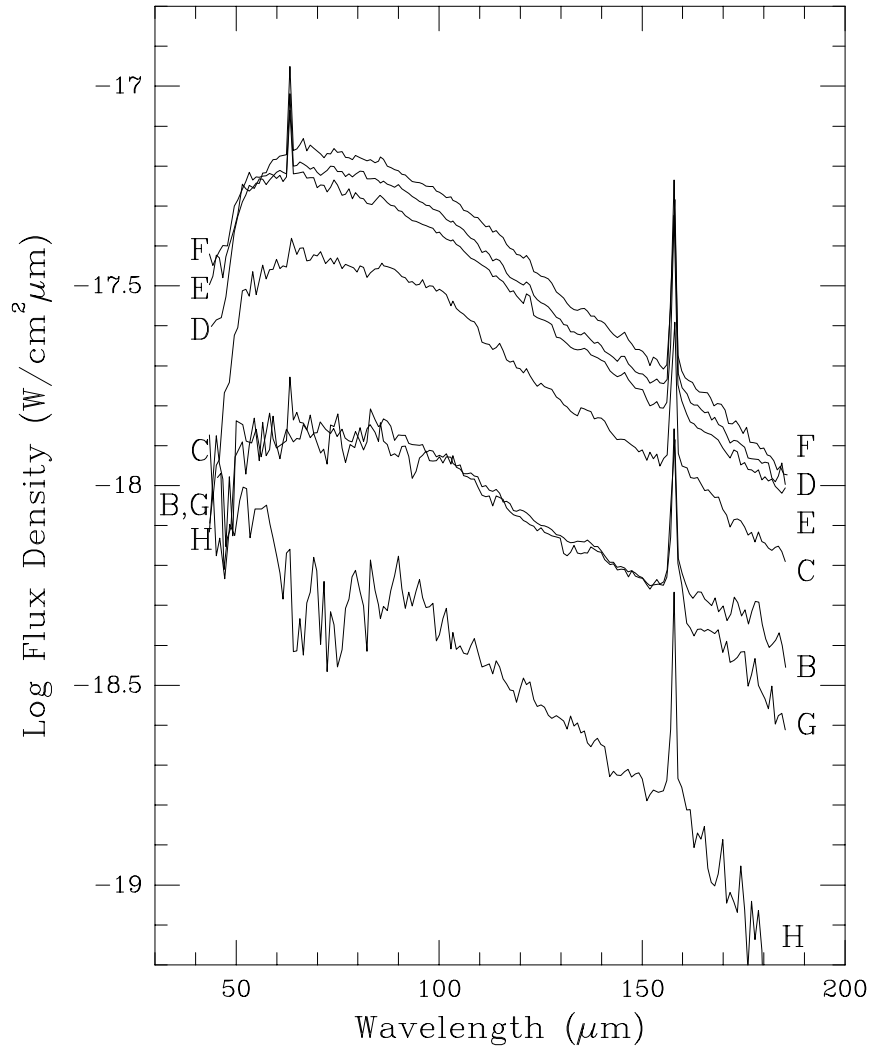

Fig. 4. LWS spectra for positions B-H in Fig. 3.

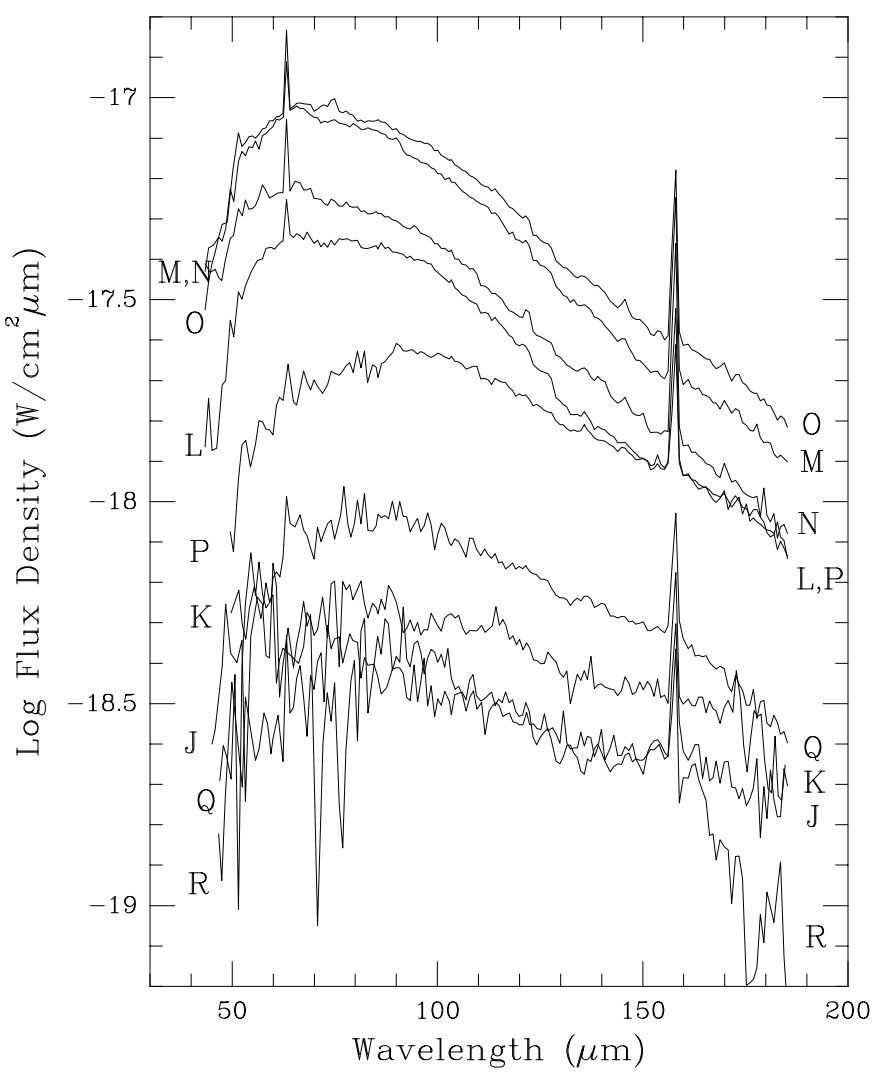

Fig. 5. LWS spectra for positions J-R in Fig. 3.

of UV radiation in the HII region. In addition, a region of radius $0.3 \mathrm{pc}$ surrounding the star is assumed "evacuated" 


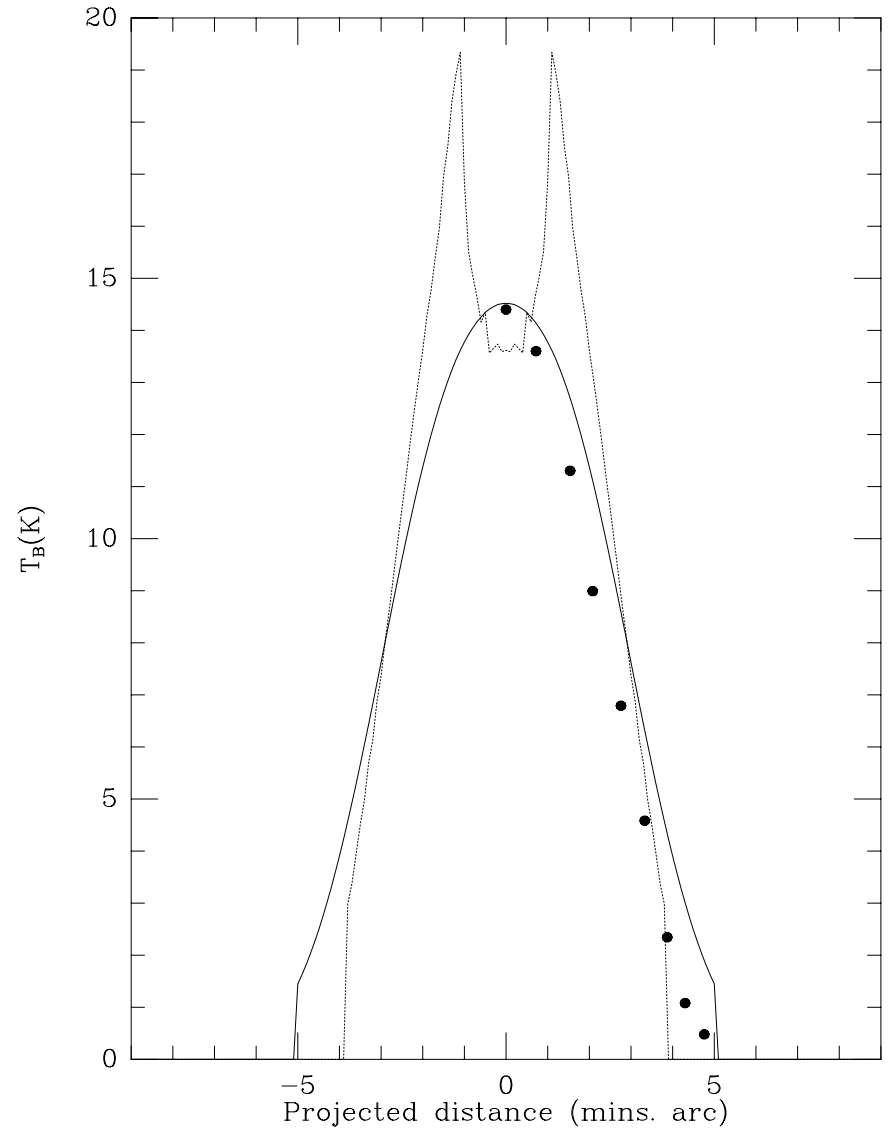

Fig. 6. The brightness temperature at $1.42 \mathrm{GHz}$ as a function of the projected distance from the ionizing star. Dotted line, model values. Solid line, model values convolved with a Gausssian profile of dispersion $1.4^{\prime}$. Solid dots, observed values as given by RI.

for gas and dust. A much smaller evacuated region would not fit the observed value of the central brightness temperature, and would put even stronger constraints on the dust/gas ratio. In the modeling by RI, the dust competed effectively for ionizing photons, leading to a low brightness temperature. However, strong dust absorption in the HII region is ruled out by the relatively cool far-infrared emission of the LWS observations.

Figure 6 shows the brightness temperature at $1.4 \mathrm{GHz}$ of this model compared to the observed values as given by RI. The radio continuum data were taken with a synthesized beam of $2.0^{\prime} \times 2.7^{\prime}$.

The solid curve shows the calculated values as convolved with a Gaussian beam of dispersion $1.4^{\prime}$. This washes out the sharp central emission drop due to the postulated "evacuated" region. The model produces a slightly wider temperature distribution than observed, but the peak value and the general shape is in fair agreement with the observations. The central emission measure is $7933 \mathrm{~cm}^{-6} \mathrm{pc}$ and compares very well to the observed value of $7930 \mathrm{~cm}^{-6} \mathrm{pc}(\mathrm{RI})$. The central HII column density is $3.2 \times 10^{20} \mathrm{~cm}^{-2}$ and the HII mass is $11 M_{\odot}$, compared to the RI values of $3.8 \times 10^{20} \mathrm{~cm}^{-2}$ and $10 M_{\odot}$, respectively. The distance from the star to the

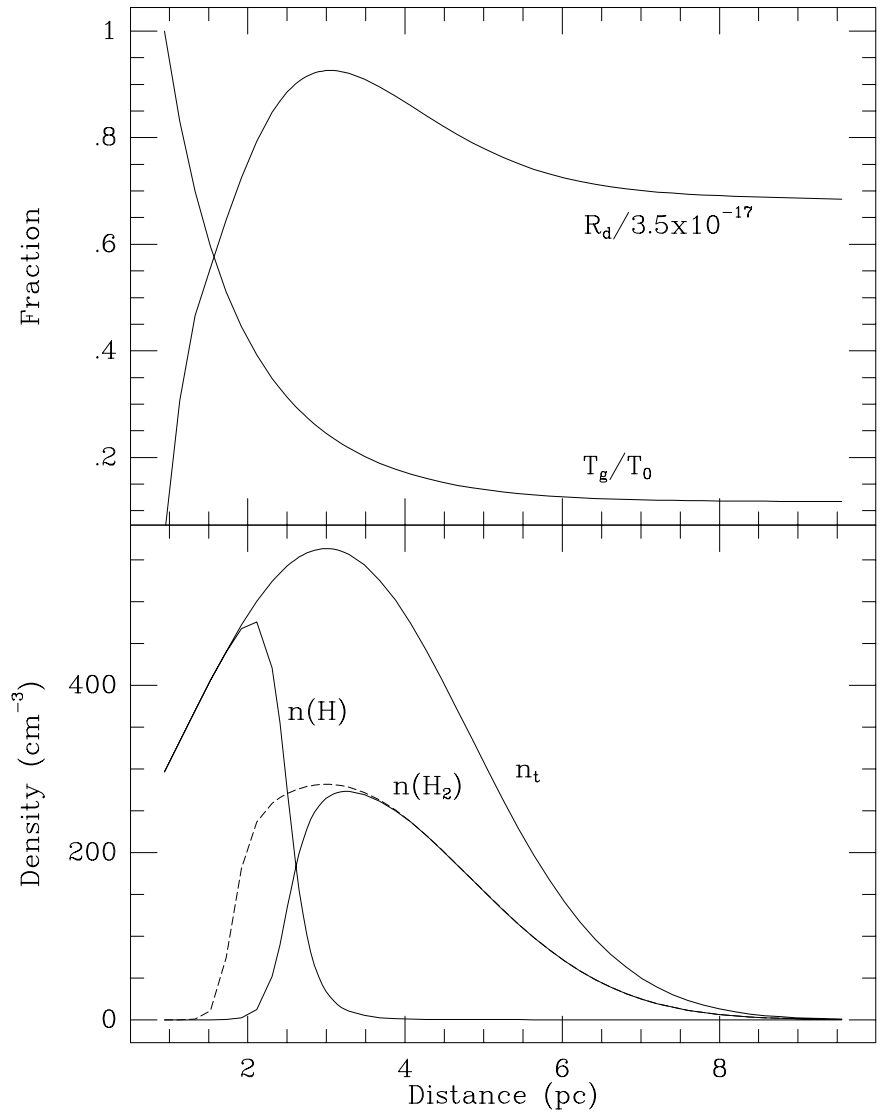

Fig. 7. Properties in the PDR as a function of distance from the star in the direction $\theta=180^{\circ}$. The silicate-fluffy graphite dust model of Fig. 2 is assumed. Upper panel: the gas temperature divided by the assumed temperature at the PDR boundary $\left(T_{0}=500 \mathrm{~K}\right)$ and the $\mathrm{H}_{2}$ formation rate coefficient divided by the constant value assumed by RI. Lower panel: the molecular hydrogen, atomic hydrogen, and total hydrogen nuclei densities. The dashed curve shows the $\mathrm{H}_{2}$ density as calculated by using RI parameters (Eq. (10)).

ionization front along $\theta=180^{\circ}$ is $0.7 \mathrm{pc}$, the same as in the RI model. In summary, the main difference between the present model of the HII region and the model presented by RI is the postulated "evacuated region" within about 0.3 pc of the star.

\subsection{Model results for the PDR}

As seen in Fig. 2, the UV opacities of the dust models are strongly wavelength-dependent and much smaller than assumed by RI. The RI values were required in order to fit the observed distribution of $\mathrm{HI}$ in the PDR. For the same reason we need to increase the relative number of small vs. large dust grains in the PDR from what is shown in Fig. 2, whilst retaining the geometry of the RI model. However, it is instructive to first consider what properties of the PDR the basic dust model would produce.

An important difference from the RI calculation is our treatment of the $\mathrm{H}_{2}$ formation rate (Sect. 3.1) which depends on the PDR dust properties and varies with the 


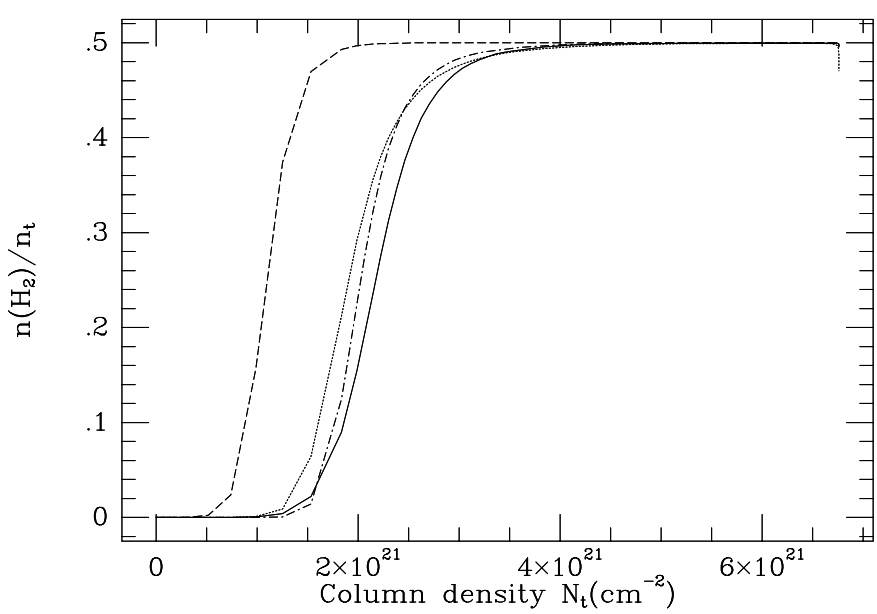

Fig. 8. The fraction of molecular hydrogen as a function of the column density of $\mathrm{H}$ nuclei in the direction $\theta=180^{\circ}$ for the same basic dust model as in Fig. 7. Solid and dash-dot curves: Fractions from Eq. (8) with the best and the simple selfshielding function, respectively. Dotted curve: fractions from Eq. (11) (constant dissociation rate). Dashed curve: fractions according to Eq. (10).

dust temperatures through the region. Figure 7 shows the $\mathrm{H}_{2}$ formation rate coefficient $R_{\mathrm{d}}$ divided by the $\mathrm{RI}$ value of $3.5 \times 10^{-17} \mathrm{~cm}^{3} \mathrm{~s}^{-1}$ as a function of the distance from the PDR boundary in the direction of $\theta=180^{\circ}$. From Eq. (4) $R_{\mathrm{d}}$ is given by

$R_{\mathrm{d}}=\sum_{i} 2.28 \times 10^{-4} a_{i, \mu \mathrm{m}}^{2} \frac{n\left(a_{i}\right)}{n_{\mathrm{t}}} S\left(T_{\mathrm{g}}\right) \gamma\left(a_{i}\right) T_{\mathrm{g}}^{1 / 2}$.

Also shown in Fig. 7 is the gas temperature $T_{\mathrm{g}}$ divided by the initial temperature $(500 \mathrm{~K})$ at the boundary. The lower panel in Fig. 7 shows the corresponding densities of atomic and molecular hydrogen, and the total density $n_{\mathrm{t}}$ of $\mathrm{H}$-nuclei. In this figure it is assumed that the dust has the properties of the fluffy dust particles. RI used the following expression for the $\mathrm{H}_{2}$ density in the PDR

$$
n_{\mathrm{H}_{2}}=\frac{R_{\mathrm{d}} n_{\mathrm{t}}^{2}}{2 R_{\mathrm{d}} n_{\mathrm{t}}+0.032 r^{-2} N_{\mathrm{H}_{2}}^{-1 / 2} \exp \left(-3.5 \times 10^{-21} N_{\mathrm{t}}\right)},
$$

where $r$ is the distance from the star (assumed B0 V) in pc, $N_{\mathrm{t}}$ is the column density of H-nuclei, and $R_{\mathrm{d}}$ is the constant value given above. The dashed curve in Fig. 7 plots this relation, and shows that our dust model gives a transition region $2 \mathrm{H} \rightarrow \mathrm{H}_{2}$ about 1 pc further into the cloud. Both a lower formation rate of $\mathrm{H}_{2}$ and a stronger radiation field (less UV absorption by dust) than assumed by RI contribute to this.

For the same dust and the same spatial direction, the solid curve in Fig. 8 shows the molecular fraction as a function of the total column density into the cloud. The dot-dashed curve shows the effect of using the simple selfshielding function (Draine \& Bertoldi 1996):

$f_{\mathrm{s}}=\left(N_{\mathrm{H}_{2}} / 10^{14}\right)^{-0.75}$ if $N_{\mathrm{H}_{2}}>10^{14} \mathrm{~cm}^{-2}$, otherwise $f_{\mathrm{s}}=1$. For the column densities of interest here $\left(10^{19} \mathrm{~cm}^{-2} \leq N_{\mathrm{H}_{2}} \leq 10^{21} \mathrm{~cm}^{-2}\right)$ the simple shielding function is numerically smaller than the more accurate function and therefore gives a transition region that is slightly closer to the star, i.e. it overestimates the $\mathrm{H}_{2}$ abundance. Also shown (dotted curve) is the molecular fraction predicted by an iterative solution of (Hollenbach \& Tielens 1999)

$4 \times 10^{-11} G_{\mathrm{uv}}(p) x f_{\mathrm{s}}\left(N_{\mathrm{H}_{2}}\right)-2(1-x) R_{\mathrm{d}} n_{\mathrm{t}}(p)=0$,

where $f_{\mathrm{s}}$ is the simple function above, $R_{\mathrm{d}}$ is at a constant value of $3 \times 10^{-17} \mathrm{~cm}^{3} \mathrm{~s}^{-1}$, and $G_{\mathrm{uv}}$ is the radiation field from 6 to $13.6 \mathrm{eV}$ in units of $1.6 \times 10^{-3} \mathrm{ergs} \mathrm{cm}^{-2} \mathrm{~s}^{-1}$. This also predicts a transition slightly closer to the star, mainly as a result of the constant and higher formation rate coefficient $R_{\mathrm{d}}$ (cf. Fig. 7) The RI result is shown by the dashed curve and gives a much closer transition region, with a correspondingly higher $\mathrm{H}_{2}$ column density of $2.9 \times$ $10^{21} \mathrm{~cm}^{-2}$ compared with $2.3 \times 10^{21} \mathrm{~cm}^{-2}$ for the solid curve. Note that this difference has a large effect on the total column density of $\mathrm{H}$-atoms, giving $N(\mathrm{H})=1.1 \times 10^{21}$ for RI versus $2.2 \times 10^{21} \mathrm{~cm}^{-2}$. However, it is the RI value that is in agreement with the observed value of the $\mathrm{HI}$ column density. Hence it is necessary in our modeling to redetermine the size distribution of the dust in the PDR so as to match the measured low HI column density.

Figure 9 shows the model calculations for the HI column density profile perpendicular to the line of sight to the ionizing star for both the "normal" dust size distribution (Sect. 4) (upper curves) and a redetermined distribution for the PDR grains with fractional abundances in the dust of $\mathrm{Si}$ and $\mathrm{C}$ equal to $(0.4,0.58)$ and $(0.25$, 0.37 ) for the (large, very small) grains, respectively (lower curves). In both cases the lower and upper large dust grain size limits are 0.0156 and $0.25 \mu \mathrm{m}$, respectively. The relative enhancement of the number of very small grains increases the absorption coefficient of $\mathrm{H}_{2}$ dissociating photons by almost a factor of 3 , or close to the value assumed by RI. The HI observations demand such an enhancement in the PDR dust absorption properties, if the dust is a silicate-carbon mixture. Small grains may be produced at the expense of larger grains by destructive processes such as shattering in turbulent motions and shock waves at the HII/PDR interface, particularly by grain-grain collisions of porous and/or fractal structure. The model is seen to reproduce the HI emission quite well, except for the outermost points, where the observations show low-level extended emission. The same result was found by RI.

\subsection{Model results for the far-IR continuum emission}

With a model that accounts for both the HII brightness temperature and the $\mathrm{HI}$ emission in the neutral region, the question is how well this model accounts for the LWS continuum spectra (Figs. 4 and 5). Assuming dust with enhanced far-IR emission properties (solid curve in Fig. 2), as well as enhanced numbers of very small grains (cf. Fig. 9), the solid curves in Fig. 10 shows the model emission integrated along the lines of sights for positions L, N, 


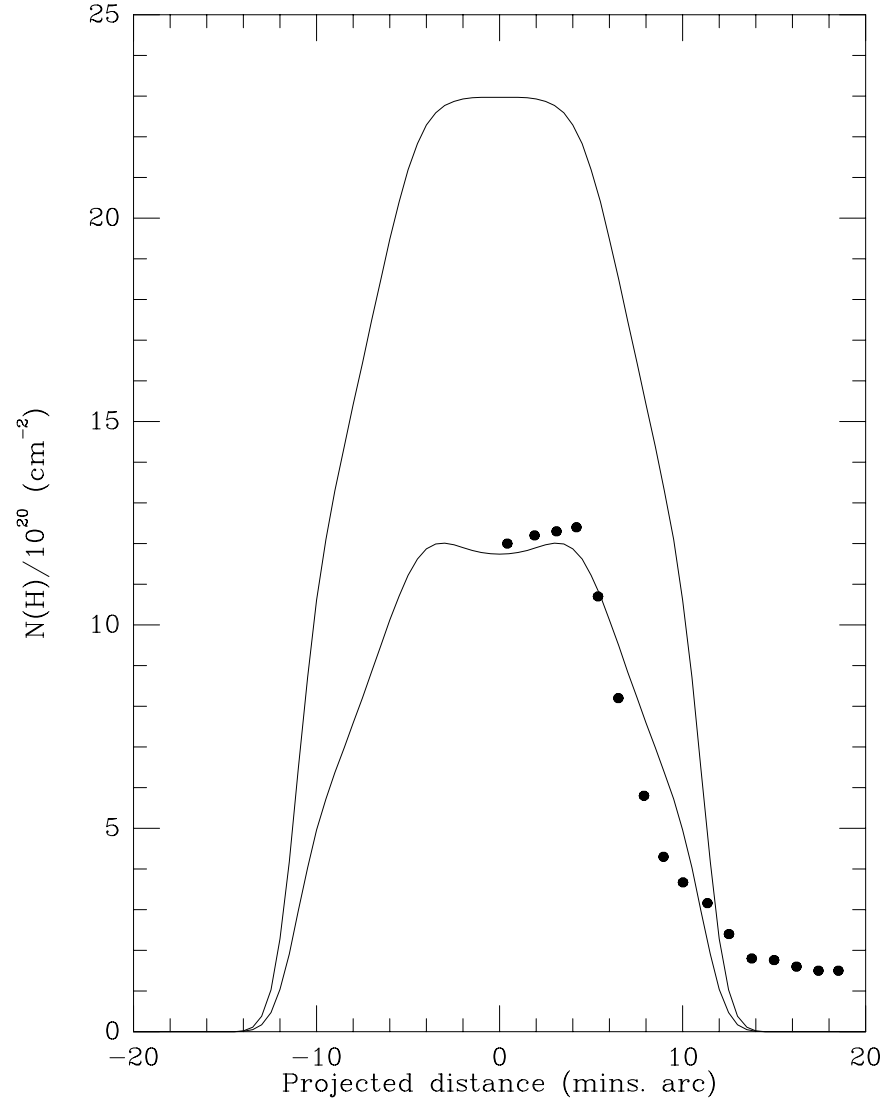

Fig. 9. The column density of $\mathrm{HI}$ as a function of the distance from the ionizing star. Solid dots: observed values as given by RI. Solid curves are model calculations convolved with a gaussian beam of dispersion $1^{\prime}$. Upper curve: "normal" dust size distributions as in Fig. 2. Lower curve: enhanced numbers of very small vs. the larger dust grains in the PDR.

$\mathrm{O}$, and $\mathrm{P}$ compared to the observed continuum. The upturn of the calculated spectra at wavelengths below about $30 \mu \mathrm{m}$ at positions $\mathrm{N}$ and $\mathrm{O}$ is due to emission from the (very depleted) hotter dust within the HII region, but this is outside the LWS wavelength range. The far-IR emission is entirely from the dust in the PDR. However, even in this case of enhanced emissivity, the peaks of the observed spectra are at longer wavelengths and at higher levels than the model emission. Postulating much larger grains in the PDR does not improve the situation, since the HI observations require the presence of small grains. In fact, a model calculation with the large grain population having sizes from 0.25 to $0.93 \mu \mathrm{m}$, moves the peak emission to slightly shorter wavelengths, owing to an increase in the very small grain population required to still account for the HI emission profile.

Only dust with significantly increased infra-red emissivities could conceivably have a peak emission as shown in the LWS observations, and we therefore consider emission by the fractal grains and by the ellipsoidal grains (cf. Fig. 2). Since the UV properties of these grains are the same as for the basic silicate-graphite model, we assume again an enhanced presence of the very small grains, so as to get agreement with the HI profile. The model

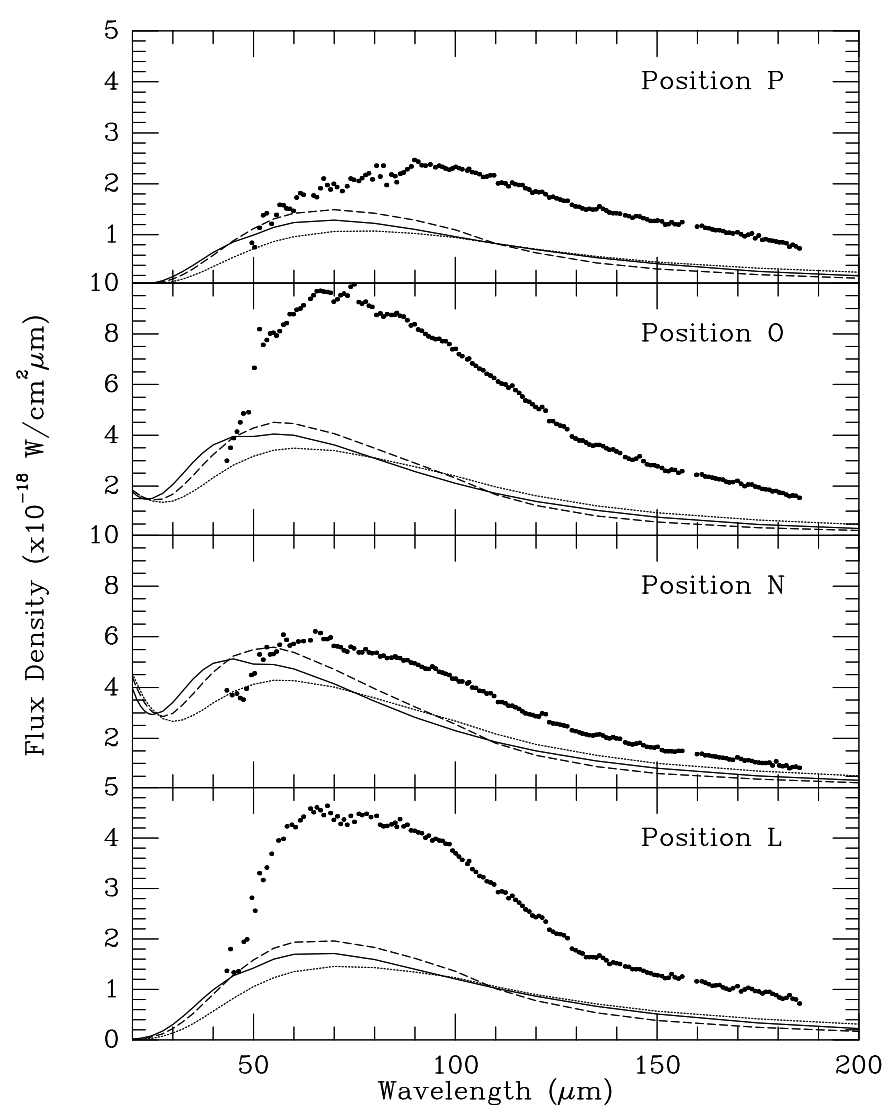

Fig. 10. The RI model continuum emission compared to observed LWS spectra (with line emission removed) for positions in the scan of Fig. 3. Solid lines, emission from spherical silicate-graphite dust with enhanced far-IR emissivity. Dashed lines, emission from fractal silicate-graphite dust. Dotted lines, emission from ellipsoidal silicate-graphite dust.

emission from the fractal and ellipsoidal grains are shown in Fig. 10 by the dashed and dotted curves, respectively. As expected, the emission now peaks at slightly longer wavelengths, but the shift is not enough for them to match the LWS data. Also, although the level of peak emission is broadly compatible with the observations for the central position $(\mathrm{N})$, the predicted levels are too low by factors of 2-3 for the other positions in Fig. 10. We conclude that the RI model cannot produce agreement with both the HI and the LWS data, even when fairly extreme dust emissivities are assumed. A density distribution less centrally condensed than the Gaussian distribution of RI may provide a better fit to the infrared data. Below we subject a "blister model" to the same constraints as the RI model.

\section{Model using a blister geometry}

The density distribution in this model is axisymmetric and given by

$n(r, \theta)=n_{0} \mathrm{e}^{-r \cos \theta / h}$ for $r \geq r_{0}(\theta)$,

$n(r, \theta)=0$ for $r<r_{0}(\theta), \quad$ where

$r_{0}(\theta)=R_{0} \mathrm{e}^{\cos \theta / h_{0}}$. 

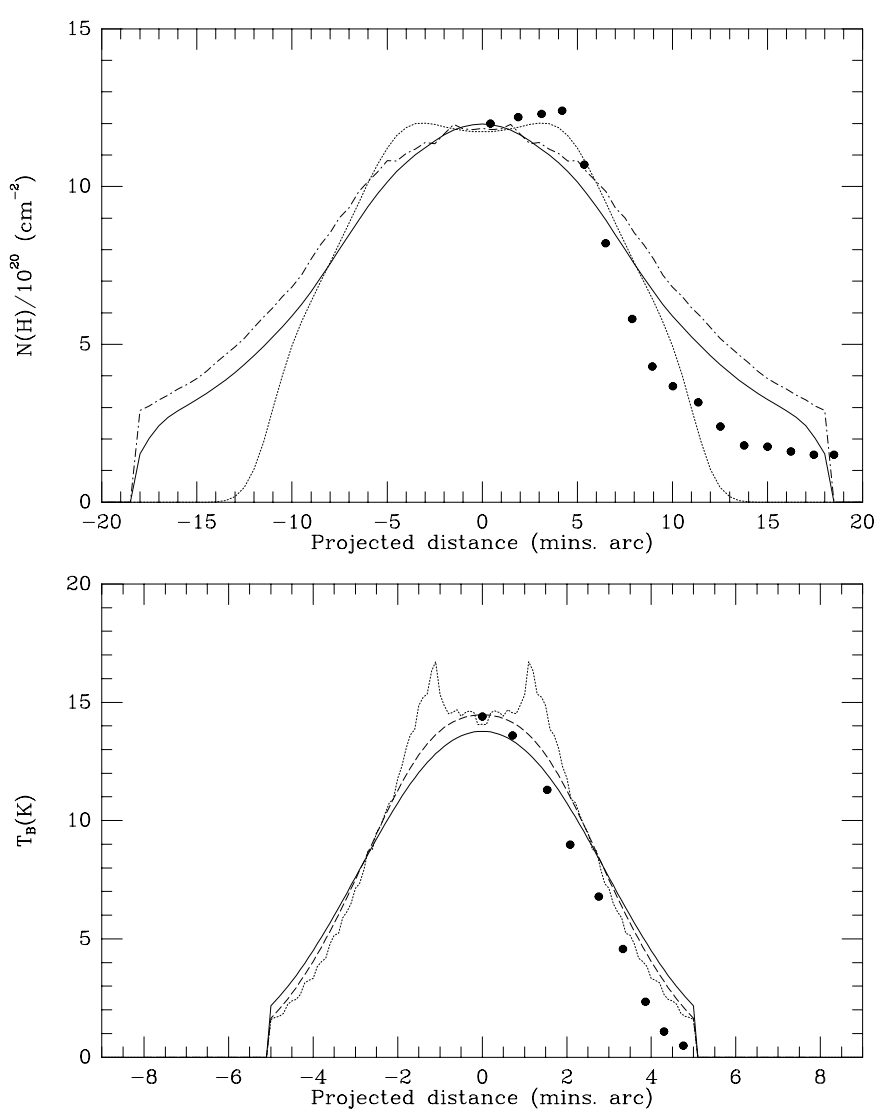

Fig. 11. Upper panel: the column density of HI as a function of distance from the ionizing star. Solid dots: observed values as given by RI. Solid curve and dash-dot curve: model calculations for the blister model convolved with Gaussian beam of dispersion $1^{\prime}$ for a scale height of 0.65 and $0.8 \mathrm{pc}$, respectively. Dotted curve: the RI model curve from Fig. 9. Lower panel: the brightness temperature at $1.42 \mathrm{GHz}$ as a function of the projected distance from the ionizing star. Solid and dashed curves, blister model calculations as convolved with Gaussian beams of dispersion $1.4^{\prime}$ and $1.0^{\prime}$, respectively.

The "evacuated" region may also have an axisymmetric shape, but we here assume a spherical shape by setting the numerical factor $h_{0}=100$. The value of $R_{0}=0.3 \mathrm{pc}$, as for the RI model.

Figure 11 shows the fits to the HI and to the brightness temperature data. The density parameter $\left(n_{0}=60 \mathrm{~cm}^{-3}\right)$, the scale height $(h=0.65,0.8 \mathrm{pc})$, and the relative dust fractions have been chosen so as to give a good fit to the central value of the HI emission profile, as shown in the upper panel of Fig. 11. For the curve shown (spherical silicate-graphite dust) the $\mathrm{Si}$ and $\mathrm{C}$ fractional abundances are $(0.42,0.56)$ and $(0.27,0.35)$ for the (large, very small) grains, respectively, or almost identical to the values for the RI model. The other grain models give very similar fractional abundances. The dust size limits in this model differ from the RI model calculations only in that the lower limit is $0.058 \mu \mathrm{m}$ rather than $0.0156 \mu \mathrm{m}$.

The width of the computed profile in Fig. 11 is broader than the observed width, but any smaller value of the scale height results in hotter grains, giving even more far

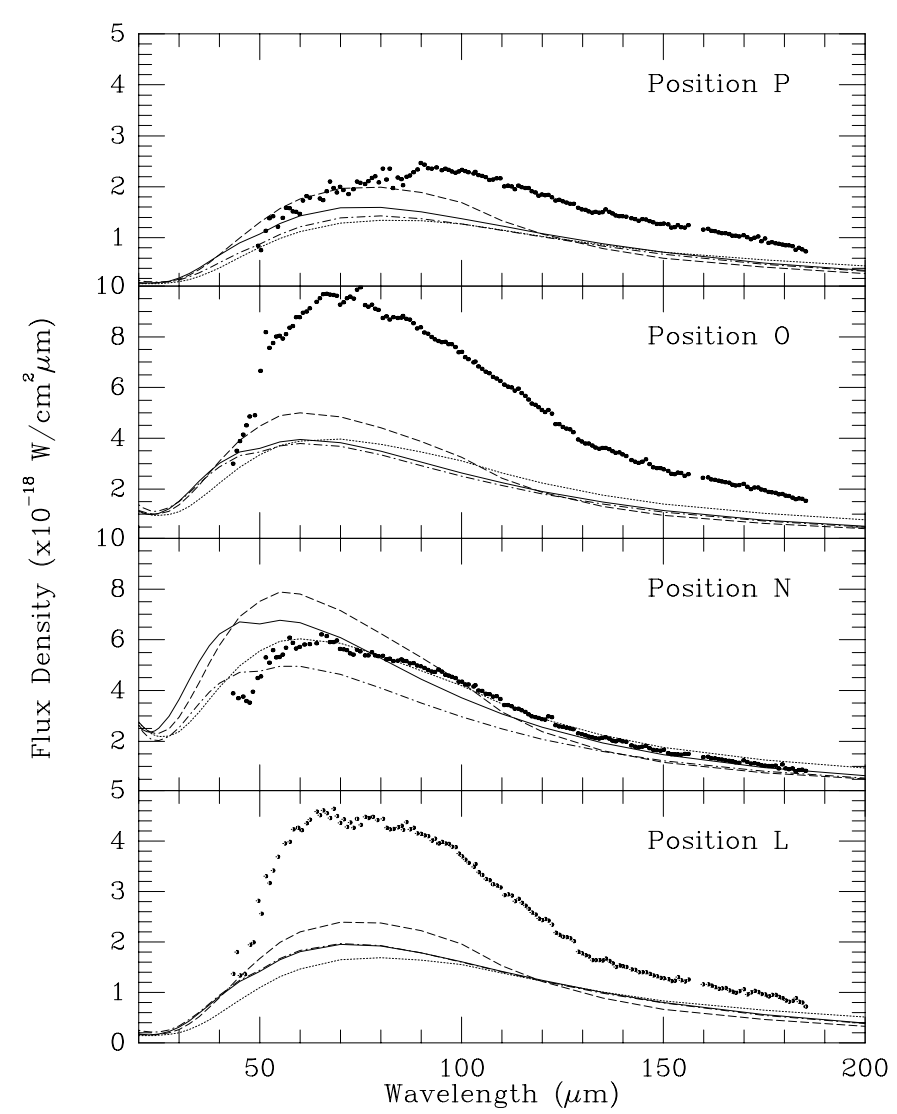

Fig. 12. The blister model continuum emission compared to observed LWS spectra (with line emission removed) for positions in the scan of Fig. 3. Solid lines, emission from spherical silicate-graphite dust with enhanced far-IR emissivity. Dashed lines, emission from fractal silicate-graphite dust. Dotted lines, emission from ellipsoidal silicate-graphite dust. For position N, the dash-dot curve shows the spectrum for a scale height of $0.8 \mathrm{pc}$, rather than $0.65 \mathrm{pc}$ as for the other curves.

infra-red emission, which already exceeds the observed level in position $\mathrm{N}$ (Fig. 12). For the far-infrared dust emission to be consistent with the observations requires an increase in the scale height from $0.65 \mathrm{pc}$ to $0.8 \mathrm{pc}$, with a corresponding slight increase in the HI profile width (dash-dot curve in Fig. 11). We note that the blister model does not show the slight minimum in $N(\mathrm{H})$ at the central position, as observed and as reproduced in the RI model (dotted curve). On the other hand, it gives more emission in the outer parts, where the RI model falls short of the observations. Although adequate for our present purposes, the modeling shown by the RI and blister geometries appears to bracket the observed HI profile width, indicating that a density distribution intermediate between these two would give an even better fit.

The $1.42 \mathrm{GHz}$ brightness temperature profile produced by the blister model is shown in the lower panel of Fig. 11, and is slightly broader than the observed profile, as was also the case for the RI model (Fig. 6). The dashed curve shows the result for a convolution with a narrower Gaussian beam of dispersion 1.0', fitting the data slightly better. The distance between the star and the 
ionization front in the $\theta=180^{\circ}$ direction is $0.8 \mathrm{pc}$, slightly longer then in the RI model. The emission measure is $8200 \mathrm{~cm}^{-6} \mathrm{pc}$, again close to the observed values, but the total HII mass is about $22 M_{\odot}$, or twice the value in the RI model.

The emission spectra for the three different dust emission properties are shown in Fig. 12. As expected, the blister model emissions peak at longer wavelengths than the case for the RI model, but still do not match the observed emission peaks, except for position L. In addition, as for the RI model (Fig. 10), the level of emission is too low in comparison to the data, except for the central position $\mathrm{N}$. At $\mathrm{N}$, for a scale height of $0.65 \mathrm{pc}$, the predicted level of emission is too high at the shorter wavelengths, except in the case of the ellipsoidal grains, which have the highest far-infrared emissivities (cf. Fig. 2). A larger value for the scale height of $0.8 \mathrm{pc}$ does not violate the observed level of emission (dash-dot curve), but, as already noted, gives a broader HI profile than observed (Fig. 11, upper panel).

\section{Discussion}

\subsection{The models}

In our model calculations we have explored the outputs for variations in the input parameters, and find quite closely constrained values. Specifically, it was found necessary to model an evacuated region around the exciting star, as well as a small dust/gas ratio in the remainder of the HII region. A dust- and/or gas-depleted region may form around a massive star by radiation pressure on the dust before the star ionizes the remaining gas or by the action of a later stellar wind. While dust free gas is expected in compact HII regions, and strong dust depletion is implied by the modeling of several regions (Chini et al. 1986; Churchwell et al. 1990), it is surprising to find strong dust depletion in a low-density nebula such as S125. On the other hand, a champagne phase leading to a blister region may transform a compact region quite "suddenly" to an extended low-density region (Yorke 1986).

The results of the model calculations show that neither the off-set spherically symmetric density distribution of the RI model or the axisymmetric blister density distribution can account simultaneously for the HI, HII and far-infrared data. Constrained by the need for many small dust grains in the PDR to explain the HI profile, the computed far-infrared spectra peak at slightly shorter wavelengths than the observed spectra and do not give high enough levels of emission at all but the central point of the LWS scans. The excess emission computed at position N (Fig. 12) may imply that the increase in the number of small dust grains outside the HII boundary is gradual, and not abrupt, as is assumed here. However, a main result of these calculations is that neither density distribution model can explain the much higher levels of emission observed in the symmetric positions $\mathrm{M}$ and $\mathrm{O}, \mathrm{D}$ and $\mathrm{F}$ compared to the central postions $\mathrm{E}$ and $\mathrm{N}$ (Figs. 4 and 5). For the parameters of the models giving
HII brightness temperature and HI column density profiles that do agree reasonably well with observations, the computed far-infrared emission levels decrease monotonically away from the central position. It appears necessary to require an additional component of dust emission. This must be dust associated with molecular cloud material, since there is no corresponding signature in the HI data. The maps by Sargent et al. (1981) at $85 \mu \mathrm{m}$ and $150 \mu \mathrm{m}$ showed far-infrared peaks away from the ionizing star, and they suggested an embedded object to the south-east, designated IC 5146FIR, which is close to our position O where the maximum amount of emission occurs. Their two secondary peaks are close to our positions $\mathrm{D}$ and $\mathrm{F}$ which are also the positions of maximum emission in the A-I scan. However, the LWS spectra show almost as much emission at $\mathrm{M}$ as at $\mathrm{O}$ (Fig. 5), indicating a quite symmetric "ring" of additional far-infrared emission in S125. Three regions of enhanced $\mathrm{CO}$ emission lie around the periphery of S125 (McCutcheon et al. 1982; Lada \& Elmegreen 1979), but, except for IC 5146IR, do not coincide with the FIR peaks. The symmetry of the emission seen in the LWS scans implies an association with the geometry of the S125 region, and our modeling suggests the need for embedded sources of emission in a region surrounding S125. Dobashi et al. (1992) noted that six IRAS sources were associated with their observed $\mathrm{CO}$ emission in this region.

A possible other source of additional far-infrared emission is reabsorption of the near infra-red emission (10-20 $\mu \mathrm{m})$, with subsequent re-emission at longer wavelenths, which has not been taken into account here. However, the positions $\mathrm{M}$ and $\mathrm{O}, \mathrm{D}$ and $\mathrm{F}$ are at a projected distance of only $3^{\prime}$, or $0.8 \mathrm{pc}$ at the distance of S125. This is of the order of only 1-2 scale heights in the blister models for $\theta=90^{\circ}-135^{\circ}$, while the 10 micron optical depth does not reach 0.5 until several $(\geq 5)$ scale heights. The fraction of energy in the near-infrared region is also inadequate to raise the far-infrared emission by up to $100 \%$, as in position $\mathrm{O}$.

It seems that the simplest modification to the present modeling is to assume the presence of additional (molecular) material connected with the HII region, and with additional (and unknown) sources of radiative energy. Since IRAS maps and radio observations strongly suggest there are embedded sources in the molecular cloud, it is important to identify how much of the emission from these regions can be attributed to the central star, in order to separate out the emission from the embedded sources. By using the model emissions calculated here which fit the HI and HII data, we attempt below to characterize this far-infrared emission component.

\subsection{The additional component}

The additional far-infrared emission is parameterized by the column density of hydrogen nuclei $N_{\mathrm{H}}$ and an ambient radiation field as a function of the ratio $\chi$ of its energy density relative to the average interstellar radiation field 


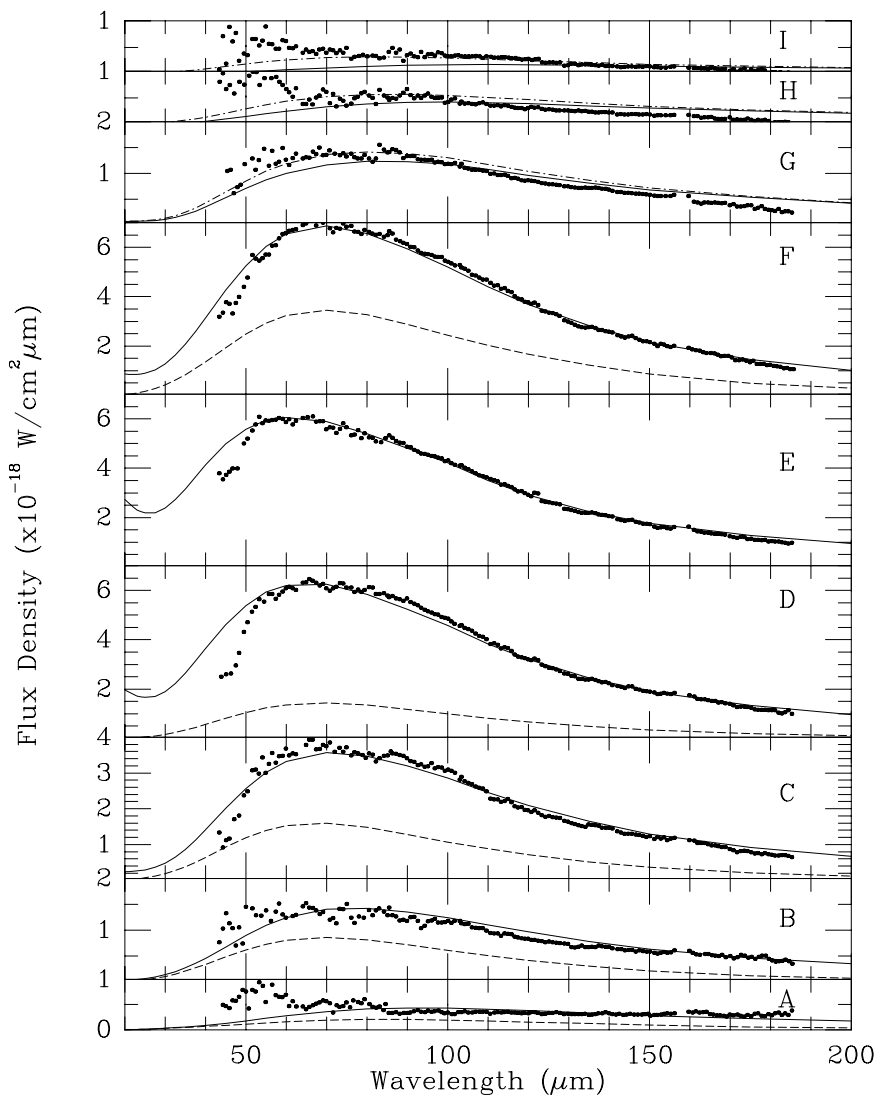

Fig. 13. Fits to the observed LWS spectra (with line emission removed) for all the positions in the scan A-I of Fig. 3. Solid lines, emission from the S125 model (assuming ellipsoidal silicate-graphite dust) + emission from the postulated additional component. The additional emission, with parameters as given in Table 1, is shown by the dashed lines. For positions G, H, and I the dash-dot curves show the fits for an increased value of the radiation field.

energy density of $0.7 \mathrm{eV} / \mathrm{cm}^{3}$ (cf. Guhathakurta \& Draine 1989). The column density scales the emission level, while the value of $\chi$ determines the wavelengths of peak emission. We assume that the dust composition, abundances, size distributions, and far-infrared emissivities in this component are given by the basic dust model discussed in Sect. 4, i.e. the dust is the silicate-graphite model consistent with a normal interstellar extinction curve. Thermal fluctuations are included in calculating the emission from the very small dust grains of radius $0.005 \mu \mathrm{m}$, following the procedure by Guhathakurta \& Draine (1989). However, no enhancements of far-infrared emissivities are assumed for this dust component.

For the HII region +PDR model emission, we shall use the blister model emission by the ellipsoidal silicategraphite dust (dotted curves in Fig. 12). This is the only dust model that does not exceed the observed levels of emission at any of the observed positions. However, with the exception of the central positions $\mathrm{N}$ and $\mathrm{E}$, the other dust models as shown in Fig. 12 would give very similar parameter values $N_{\mathrm{H}}$ and $\chi$. Figures 13 and 14 show fits to all the LWS spectra, with the contribution by the

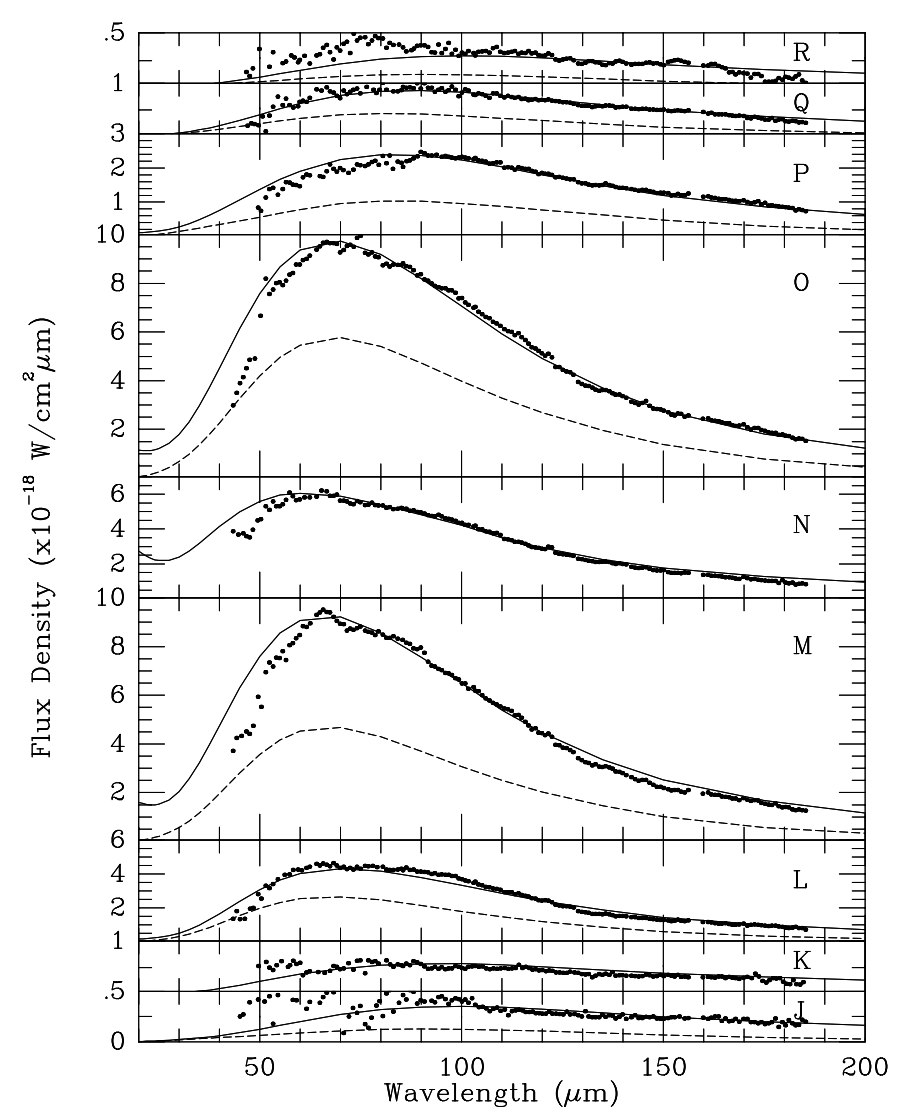

Fig. 14. Fits to the observed LWS spectra (with line emission removed) for all the positions in the scan J-R of Fig. 3. Solid lines, emission from the S125 model (assuming ellipsoidal silicate-graphite dust) + emission from the postulated additional component. The additional emission, with parameters as given in Table 1, is shown by the dashed lines.

"additional component" shown as the dashed curves. Most of the spectra can be fitted fairly well by assuming a contribution from the additional component that is very low in the center of the HII region, is large in the immedate positions surrounding the center, and then tapers off to be insignificant in the outer positions (Table 1). Most discrepancies with observed values are found at the shortest wavelengths $(\lambda \leq 60 \mu \mathrm{m})$, where the spectra are the noisiest. However, for some of the outer scan positions the emission appears to peak at the shortest wavelengths $(\lambda \leq 60 \mu \mathrm{m})$. For positions G, H, and I the dash-dot curves in Fig. 13 thus show additional fits for an increased value of $\chi$ and a decreased value of $N_{\mathrm{H}}$. Table 1 gives the parameters for each fit, and also lists the observed LWS fluxes, the ratio of total flux/observed flux, and the fractions of blister model flux/total flux.

Figure 15 shows the spatial variation of the "additional emission" at several wavelengths. For the positions G, H, and I only the first entry in Table 1 is shown. The second entries would only slightly increase the level of emission at these positions. The emission appears fairly symmetric about the central scan position, although less so in the $\mathrm{A}-\mathrm{I}$ scan compared to the J-R scan. Therefore a ring or shell structure surrounding S125 is indicated. 
Table 1. Properties for models in Figs. 13 and 14.

\begin{tabular}{cccccc}
\hline Position & $\chi^{\mathrm{a}}$ & $\begin{array}{c}N_{\mathrm{H}}^{\mathrm{b}} \\
\left(10^{21} \mathrm{~cm}^{-2}\right)\end{array}$ & $\begin{array}{c}\text { Flux }^{\mathrm{c}} \\
\left(10^{-17} \mathrm{Wcm}^{-2}\right)\end{array}$ & Ratio $^{\mathrm{d}}$ & Fraction $^{\mathrm{e}}$ \\
\hline $\mathrm{A}$ & 3 & 2 & 5.7 & 0.88 & 0.58 \\
$\mathrm{~B}$ & 10 & 2 & 13. & 1.2 & 0.49 \\
$\mathrm{C}$ & 12 & 3 & 31. & 1.1 & 0.62 \\
$\mathrm{D}$ & 11 & 3 & 52. & 1.2 & 0.80 \\
$\mathrm{E}$ & $\leq 1$ & $\leq 1$ & 49. & 1.2 & 1.0 \\
$\mathrm{~F}$ & 10 & 8 & 58. & 1.1 & 0.55 \\
$\mathrm{G}$ & $\leq 1$ & $\leq 1$ & 13. & 1.1 & 0.93 \\
& 10 & 0.5 & 13. & 1.2 & 0.87 \\
$\mathrm{H}$ & $\leq 1$ & $\leq 1$ & 5.3 & 0.94 & 0.92 \\
& 10 & 0.5 & 5.3 & 1.2 & 0.72 \\
$\mathrm{I}$ & $\leq 1$ & $\leq 1$ & 4. & 0.53 & 0.86 \\
& 10 & 0.5 & 4. & 0.90 & 0.50 \\
\hline $\mathrm{J}$ & 2 & 2 & 4.4 & 0.91 & 0.66 \\
$\mathrm{~K}$ & $\leq 1$ & $\leq 1$ & 5.8 & 1.2 & 1.0 \\
$\mathrm{~L}$ & 12 & 5 & 37. & 1.1 & 0.45 \\
$\mathrm{M}$ & 13 & 8 & 71. & 1.2 & 0.54 \\
$\mathrm{~N}$ & $\leq 1$ & $\leq 1$ & 48. & 1.2 & 1.0 \\
$\mathrm{O}$ & 11 & 12 & 79. & 1.1 & 0.46 \\
$\mathrm{P}$ & 3 & 10 & 22. & 1.1 & 0.60 \\
$\mathrm{Q}$ & 4 & 3 & 8.8 & 1.1 & 0.56 \\
$\mathrm{R}$ & 2 & 2 & 3.8 & 0.92 & 0.57 \\
\hline & & & & & \\
\hline
\end{tabular}

a The value of the radiation field energy density in the additional component relative to the average interstellar value.

b The column density of hydrogen nuclei that scales the emission from the additional component.

c The observed LWS flux (45-200 $\mu \mathrm{m})$.

d The ratio of the total model flux to the observed flux.

e The fraction of the total flux emitted by the HII+PDR model.

The intensity level of the emission increases as the wavelength decreases from 150 to $70 \mu \mathrm{m}$.

\section{Conclusions}

The main conclusions to be drawn from this modeling of S125 are

1) The observed LWS spectra require a dust-gas mass ratio within the HII region which is $\leq 10^{-6}$, i.e. it is strongly "dust-depleted". Assuming large grain sizes up to $1 \mu \mathrm{m}$ does not change this conclusion.

2) Both the off-set spherically symmetric density distribution of RI and an axisymmetric exponential density distribution provide reasonable fits to the HII brightness temperature profile and the $\mathrm{HI}$ column density profile, if it is assumed that an "evacuated" region of radius about $0.3 \mathrm{pc}$ surrounds the star.

3) However, fits are not obtained for all of the LWS farIR spectra unless additional sources of far-infrared emission are present in a roughly symmetric region around and at the periphery of the HII region.

4) The dust heated by the central star and emitting in the ionized and neutral regions must have far-infrared
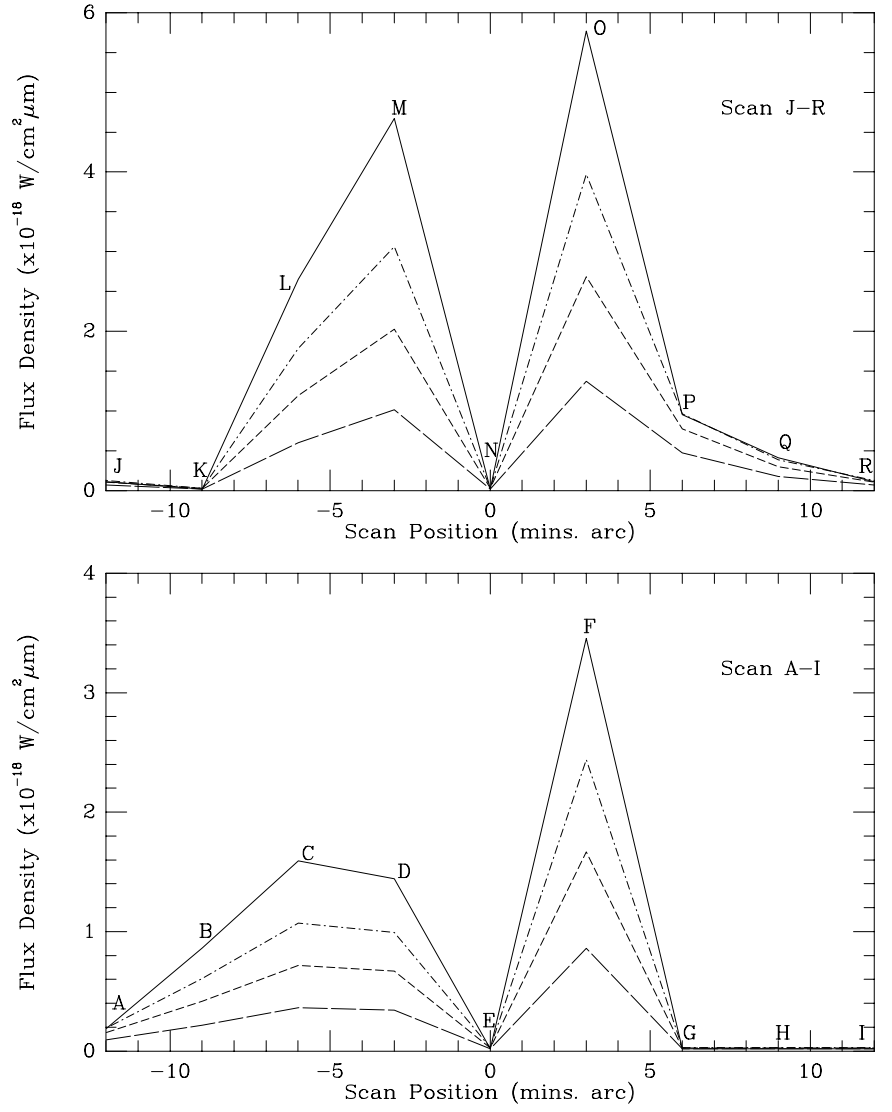

Fig. 15. Upper panel: The additional emission as a function of scan position for the scan J-R in Fig. 3. Solid curve, at $70 \mu \mathrm{m}$; dash-dot curve, at $100 \mu \mathrm{m}$; short-dash curve, at $120 \mu \mathrm{m}$; longdash curve, at $150 \mu \mathrm{m}$. Lower panel: The additional emission as a function of position for the scan A-I in Fig. 3. Curves as in the upper panel.

emissivities greater than the "normal" silicate-graphite dust, as a consequence of composition, shape, or porosity.

5) The contribution from the HII region as powered by the central star BD $+46^{\circ} 3474$ can account for about $50-100 \%$ of the $45-200 \mu \mathrm{m}$ emission in the 17 positions observed, with maximum contribution in the center and far from the center.

6) The scale height in the exponential "blister" model is $0.65-0.8 \mathrm{pc}$, and the density parameter $n_{0} \simeq 60 \mathrm{~cm}^{-3}$.

7) The dust-gas mass ratio in the PDR is normal, $\leq 10^{-2}$, but a major fraction of the assumed silicategraphite dust is required to be present in the very small grain components $(\sim 0.005 \mu \mathrm{m})$ in order to explain the HI column densities.

8) The detailed nature and importance of the very small grain components in S125 must await an analysis of the SWS observations in addition to the data considered here.

Acknowledgements. A portion of this work was funded by NATO Collaborative Research Grant No. 960326. 


\section{References}

Aannestad, P. A. 1989, ApJ, 338, 162

Aannestad, P. A., \& Emery, R. J. 1986, A\&A, 161, 347

Arnaud, M., \& Rothenflug, R. 1985, A\&AS, 60, 425

Aufdenberg, J. P., Hauschildt, P. H., \& Baron, E. 1999, MNRAS, 302, 599

Bazell, D., \& Dwek, E. 1990, ApJ, 360, 142

Bertoldi, F., \& Draine, B. T. 1996, ApJ, 458, 222

Biham, O., Furman, I., Katz, N., Pirronello, V., \& Vidali, G. 1998, MNRAS, 296, 869

Bohren, C. F., \& Huffman, D. R. 1983, Absorption and Scattering of Light by Small Particles (New York: WileyInterscience)

Butler, S. E., \& Dalgarno, A. 1980, ApJ, 241, 765

Cardelli, J. A., Meyer, D. N., Jura, M., \& Savage, B. D. 1996, ApJ, 467, 334

Chini, R., Krügel, E., \& Kreysa, E. 1986, A\&A, 167, 315

Churchwell, E., Wolfire, M. G., \& Wood, D. O. S. 1990, ApJ, 354,247

Crampton, D., \& Fisher, W. A. 1974, Publ. Dom. Ap. Obs. Victoria, 14, 283

Dobashi, K., Yonekura, Y., Mizuno, A., \& Fukui, Y. 1992, AJ, 104, 1525

Draine, B. T., \& Bertoldi, F. 1996, ApJ, 468, 269

Draine, B. T., \& Lee, H. M. 1984, ApJ, 285, 89

Edoh, O. 1983, Ph.D. Thesis, University of Arizona

Fogel, M. E., \& Leung, C. M. 1998, ApJ, 501, 175

Gies, D. R., \& Lambert, D. L. 1992, ApJ, 387, 673

Grevesse, N., \& Sauval, A. J. 1998, Space. Sci. Rev., 85, 161

Guhathakurta, P., \& Draine, B. T. 1989, ApJ, 345, 230

Hanner, M. S. 1987, in Infrared Observations of Comets Halley and Wilson and Properties of the Grains, NASA CP3004, 22
Hollenbach, D. J., \& Tielens, A. G. G. M. 1999, Rev. Mod. Phys., 71, 173

Hollenbach, D., \& Salpeter, E. E. 1971, ApJ, 163, 155

Kingdon, J. B., \& Ferland, G. J. 1996, ApJS, 106, 205

Kurucz, R. L. 1993, in Light Curve Modeling of Eclipsing Binary Stars, ed. E. F. Milone (Berlin: Springer)

Lada, C. J., \& Elmegreen, B. G. 1979, AJ, 84, 336

Leitch-Devlin, M. A., \& Williams, D. A. 1985, MNRAS, 213, 295

Levenson, N. A., Graham, J. R., McLean, I. S., et al. 2000, ApJ, 533, L53

Li, A., \& Draine, B. T. 2001, ApJ, 554, 778

Mathis, J. S. 1996, ApJ, 472, 643

Mathis, J. S., Rumpl, W., \& Nordsieck, K. H. 1977, ApJ, 217, 425

McCutcheon, W. H., Roger, R. S., \& Dickman, R. L. 1982, ApJ, 256, 139

Pirronello, V., Liu, C., Roser, J. E., \& Vidali, G. 1999, A\&A, 344,681

Pollack, J. P., Hollenbach, D., Beckwith, S., et al. 1994, ApJ, 421,615

Roger, R. S., \& Irwin, J. A. 1982, ApJ, 256, 127 (RI)

Sargent, A. I., van Duinen, R. J., Fridlund, C. V. M., Nordh, H. L., \& Aalders, J. W. G. 1981, ApJ, 249, 607

Savage, B. D., \& Mathis, J. S. 1979, ARA\&A, 17, 73

Sofia, U., Cardelli, J. A., Guerin, K. P., \& Meyer, D. M. 1997, ApJ, 482, L105

Verner, D. A., Ferland, G. J., Korista, K. T., \& Yakovlev, D. G. 1996, ApJ, 465, 487

Weintraub, D. A., Sandell, G., \& Duncan, W. D. 1991, ApJ, 382,270

Yorke, H. W. 1986, ARA\&A, 24, 49 